\title{
EFFECT OF SHADING BASED ON FUNCTIONAL LEAF SATURATED LIGHT INTENSITY ON THE YIELD AND WATER CONSUMPTION OF WINTER WHEAT (TRITICUM AESTIVUM L.)
}

\author{
SHEN, Y. B. - ZHANG, J. F. - LI, T.* \\ State Key Laboratory of Eco-hydraulics in Northwest Arid Region, Xi'an University of \\ Technology, Xi'an 710048, China \\ (phone: +86-029-8231-2768) \\ *Corresponding author \\ e-mail:litao@xaut.edu.cn \\ (Received $19^{\text {th }}$ Apr 2021; accepted $10^{\text {th }}$ Jun 2021)
}

\begin{abstract}
This study investigated diminished sunshine impact on crop growth and water consumption. A two-year pot experiment was conducted using the winter wheat variety "XINONG 979". Starting from the jointing stage, a control and four shading treatments were established based on the leaf saturation light intensity (LSP) of a fully light exposed functional leaf in each growth stage: (1) LSP - 500 (S1); (2) LSP -200 (S2); (3) LSP + 100 (S3); (4) LSP + $400 \mu \mathrm{mol} \mathrm{m}^{-2} \mathrm{~s}^{-1}$ (S4), and; (5) control (CK) without shading. Diurnal gas exchange and evapotranspiration were measured since jointing stage. S2, S3, and S4 showed an increase in yield and water use efficiency (WUE) under comparison to CK, with the increase in S3 being significant. Evapotranspiration in S4 increased whereas it significantly decreased in other treatments. The daily evapotranspiration of treatment S4 increased after heading to flowering stage. The net photosynthetic rate, stomatal conductance, and transpiration rate of the functional leaves of S4 showed increases at different times. The instantaneous and intrinsic WUE of functional leaves increased between 12:00-14:00. There were different shading effects on the functional leaves, whole wheat water consumption, and WUE between different shading treatments.
\end{abstract}

Keywords: water use efficiency, stomatal conductance, net photosynthetic rate, intrinsic water use efficiency, instantaneous water use efficiency, transpiration rate

\section{Introduction}

Cereals provide over $20 \%$ of carbohydrate and protein consumed internationally (Wang et al., 2005). Wheat (Triticum aestivum L.) is an essential cereal crop being globally and is cultivated widely in northern China, with the yield of this crop critical for maintaining food security in China (Dong et al., 2019).

Light, temperature, and humidity are all important factors influencing plant growth processes. Light is important for plant photosynthesis and also influences various plant macromolecule content and quality through the formation and transportation of photosynthetic products (Goto et al., 1993; Chen et al., 2019; Ohashi-Kaneko et al., 2006). Light intensity is the environmental factor most affecting crop growth and yield. However, rapid economic growth and accelerated urbanization have increased automobile and industrial pollutant emissions (Chen et al., 2019). As a result, incidents of smog have increased by an average of $16.7 \%$ to $33.3 \%$ over the past 30 years (Dong et al., 2014), which has led to a significant reduction in visibility (Ramanathan and Feng, 2009). The increased atmospheric pollution has reduced incident solar radiation by $10 \%$ to $90 \%$ (Dong et al., 2014). Several studies have revealed that solar decrease reduces leaf area index (LAI) and net photosynthesis rate $\left(\mathrm{P}_{\mathrm{n}}\right)$ in wheat leaves. Shading applied during any developmental stage significantly impairs grain filling condition, quality formation, and yield (Thangaraj and Sivasubramanian, 1990; Deng et al., 2009; 
Acreche et al., 2009; $\mathrm{Mu}$ et al., 2010). The reduction in winter wheat yield resulting from a $10 \%$ reduction in radiation may exceed 10\% (Bian et al., 2016; Mo et al., 2015). Therefore, there is an increasing need to study changes in winter wheat yield under shading.

There have been many studies on the impacts of shading on photosynthetic characteristics, the accumulation of crop dry matter, and crop yield, focusing on the adverse aspects. These include studies on the effect of lowered chlorophyll a, chlorophyll b, and non-photochemical burst coefficient (NPQ) on the use efficiency of solar energy by winter wheat leaves (Jin et al., 2018; Kromdijk et al., 2016). Other studies have found that reductions in stomatal conductance, leaf temperature, and transpiration rate resulting from shading reduced the rate of photosynthesis for wheat leaves (Dong et al., 2019; Wang et al., 2020; Xu et al., 2013). Stress from shading also leads to changes in the accumulation and distribution of plant carbohydrates, resulting in an imbalance in carbon metabolism and ultimately a reduction in yield (Grashoff and d'Antuono, 1997; Mu et al., 2010; Wang et al., 2020). There have been several studies addressing the effects of reduced sunshine on crop yield and quality in the Yangtze River Basin and the Huang-Huai-Hai region (Ili and Fallik, 2017; Mo et al., 2015; Sandri et al., 2003), with some of these studies identifying a reduction in grain yield (Gill et al., 2009).

A decrease in radiation does not necessarily have a negative effect on crop yield, particularly in areas experiencing high levels of it. Photosynthesis no longer increases with increasing radiation intensity after the photosynthesis of $\mathrm{C} 3$ crop leaves reaches the light saturation point. Excess radiation beyond the light absorption capacity of the leaves results in photoprotection and the dissipation of radiation in the form of heat during which water consumption increases. High light intensity in fruit trees increases the temperature and transpiration rate of leaves and fruits (Ho and White, 2005; Loveys and Düring, 1984; de Freitas et al., 2013). The high light intensity can increase the vapor pressure deficit (VPD) and enhance leaf transpiration of winter wheat at a rate exceeding that of fruit (Araki et al., 2000; Freitas et al., 2011; Taylor and Locascio, 2004). Studies in recent years have shown that a drop in the natural intensity of light by $22 \%$ results in increased yield due to an increased leaf area index and increased leaf light absorption because of extended growth of leaves (Mu et al., 2010; Xu et al., 2016). However, research on the effect of shading on crop water consumption, regardless of photosynthetic products and increases or decreases in yield, remains scarce.

Leaf-scale water use efficiency (WUE) provides a more direct reflection of real-time photosynthetic efficiency. Leaf intrinsic WUE $\left(\mathrm{WUE}_{\text {int }}\right)=\mathrm{P}_{\mathrm{n}} / \mathrm{g}_{\mathrm{s}}$ and instantaneous WUE (WUE $\left.E_{i n s}\right)=P_{n} / T r$, where $P_{n}$ is the net photosynthetic rate, $g_{s}$ is the stomatal conductance, and $\mathrm{Tr}$ is the transpiration rate. Many studies have been conducted on $W_{\text {Wins }}$ and $\mathrm{WUE}_{\text {int, }}$, with the results showing that WUE of leaf is mainly regulated by Tr and $g_{s}$ (Gago et al., 2014; Shahinnia et al., 2016; Wang, 2017). However, further study is needed to determine whether the effect of shading on each index can improve $W E_{i n t}$ and $W E_{\text {ins }}$ and how this effect differs during each growth period.

The present study attempted to address the above challenge by adopting winter wheat as a research object in Northwest China in which radiation is relatively strong. From the wheat jointing stage, various shading treatments based on the saturated light intensity of wheat functional leaves were established. The effects of shading on wheat yield, total water consumption, and WUE were examined to study changes to the photosynthesis, transpiration, stomatal conductance, and WUE of functional leaves under different 
shading conditions from stem elongation stage (BBCH30) to the development of fruit stage (BBCH79). The objective of this study was to clarify the effects various levels of shading have on the yield and WUE of a winter wheat variety, and thus to predict the changes the rising smog levels may induce.

\section{Materials and methods}

\section{Experimental site}

The experiments in pots were implemented during the growing seasons of winter wheat in 2016-2017 and 2017-2018 at the Xi'an University of Technology experimental site, Xi'an, Shaanxi Province, China. The site falls within $108^{\circ} 93^{\prime} \mathrm{E}, 34^{\circ}$ $23^{\prime} \mathrm{N}$ with an elevation of $416 \mathrm{~m}$. The soil type of the experiment site is a loam containing organic nitrogen, total nitrogen, available phosphorus, and available potassium of $10.0 \mathrm{~g} \mathrm{~kg}^{-1}, 1.30 \mathrm{~g} \mathrm{~kg}^{-1}, 22.3 \mathrm{mg} \mathrm{kg}$, and $110.51 \mathrm{mg} \mathrm{kg}^{-1}$, respectively. The bulk density of soil at the experiment site is $1.41 \mathrm{~g} \mathrm{~cm}^{-3}$ whereas soil $\mathrm{pH}$ is 8.03 . Figure 1 shows the meteorological data measured.

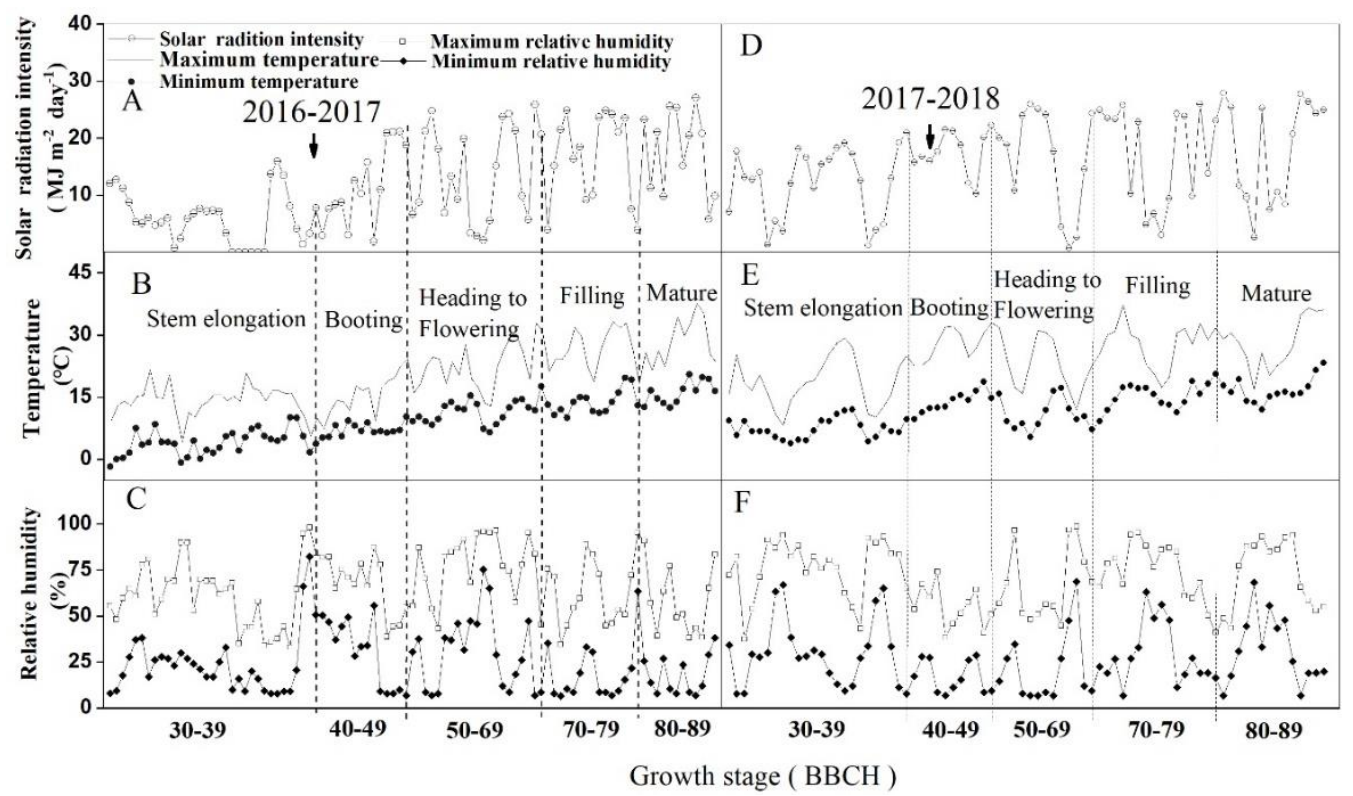

Figure 1. Meteorological data measured during a shading experiment using the winter wheat (Triticum aestivum L.) cultivar "Xinong 979". Solar radiation intensity (SRI, A, D), temperature $(T, B, E)$, relative humidity $(R H, C, F)$

\section{Experimental design and field management}

The pot experiments conducted in the current study used a winter wheat cultivar (Triticum aestivum L.) grown locally, namely "Xinong 979". White polyethylene screens containing different numbers of needles and layers were positioned $20 \mathrm{~cm}$ above the canopy of the wheat plants from the BBCH30 stem elongation stage $\left(13^{\text {th }}\right.$ February, 2017, $11^{\text {th }}$ March, 2018) to the BBCH89 senescence stage $\left(17^{\text {th }}\right.$ May, 2017, 15 ${ }^{\text {th }}$ May, 2018). The max radiation for each treatment was obtained based on the functional leaf light saturation point (LSP) during each growth stage of winter wheat. A control and four shading treatments were established: (1) LSP $-500 \mu \mathrm{mol} \mathrm{m} \mathrm{m}^{-2} \mathrm{~s}^{-1}$ (S1); (2) LSP -200 
$\mu \mathrm{mol} \mathrm{m} \mathrm{m}^{-2} \mathrm{~s}^{-1}$ (S2); (3) LSP + $100 \mu \mathrm{mol} \mathrm{m} \mathrm{s}^{-1}$ (S3); (4) LSP + $400 \mu \mathrm{mol} \mathrm{m}^{-2} \mathrm{~s}^{-1}$ (S4), and; (5) no shading (CK). The five treatments were established with four replications for a total of 20 treatments. Table 1 shows the actual experimental design used.

Table 1. Light saturation point (LSP) and upper limit of light control over different growth stages $\left(\mu \mathrm{mol} \mathrm{m} \mathrm{m}^{-2} \mathrm{~s}^{-1}\right)$

\begin{tabular}{c|c|c|c|c|c|c|c}
\hline Year & Growth stage (BBCH) & LSP & S1 & S2 & S3 & S4 & CK \\
\hline \multirow{4}{*}{2017} & Stem elongation (30-39) & 1107.79 & 600 & 900 & 1200 & 1500 & NS \\
& Booting (41-49) & 1362.42 & 850 & 1150 & 1450 & 1750 & NS \\
& Heading to flowering (51-69) & 1270.86 & 800 & 1100 & 1400 & 1700 & NS \\
& Development of fruit to senescence (71-99) & 1267.97 & 800 & 1100 & 1400 & 1700 & NS \\
\hline \multirow{4}{*}{2018} & Stem elongation (30-39) & 1244.4 & 750 & 1050 & 1350 & 1650 & NS \\
& Booting (41-49) & 1457.6 & 950 & 1250 & 1550 & 1850 & NS \\
& Heading to flowering (51-69) & 1263.29 & 750 & 1050 & 1350 & 1650 & NS \\
& Development of fruit to senescence (71-99) & 1238.55 & 750 & 1050 & 1350 & 1650 & NS \\
\hline
\end{tabular}

CK refers to the "no shading" treatment (control), whereas S4, S3, S2, and S1 refer to light saturation point (LSP) $+400 \mu \mathrm{mol} \mathrm{m} \mathrm{m}^{-2} \mathrm{~s}^{-1}$ LSP $+100 \mu \mathrm{mol} \mathrm{m}^{-2} \mathrm{~s}^{-1}$, LSP $-200 \mu \mathrm{mol} \mathrm{m}^{-2} \mathrm{~s}^{-1}$, and LSP $500 \mu \mathrm{mol} \mathrm{m}^{-2} \mathrm{~s}^{-1}$, respectively

Each pot had a height and diameter of $0.6 \mathrm{~m}$ and $0.4 \mathrm{~m}$, respectively. The pots were positioned below a rotating rain shelter under which the angle of rotation could be adjusted to ensure indirect radiation exposure. A total of $90 \mathrm{~kg}$ of soil with a bulk density of $1.41 \mathrm{~g} \mathrm{~cm}^{-3}$ was uniformly packed in each pot. Within each pot, 50 wheat seeds were broadcast sown on the $16^{\text {th }}$ October, 2016 and $15^{\text {th }}$ October, 2017. A density of 40 seedlings per pot was achieved through thinning four weeks after sowing. Irrigation was conducted during the pot experiments to achieve a soil water content (SWC) between $65 \%-95 \%$ of field capacity (FC). All treatments were irrigated to $95 \%$ FC when the SWC of some treatments approached $65 \%$ of FC. The pot and its contents were weighed to maintain the SWC. Base fertilizer in the form of $\mathrm{N}, \mathrm{P}_{2} \mathrm{O}_{5}$, and $\mathrm{K}_{2} \mathrm{O}$ were applied at 220,150, and $150 \mathrm{~kg} \mathrm{ha}^{-1}$, respectively. During the wintering period, a top dressing of $75 \mathrm{~kg} \mathrm{ha}^{-1} \mathrm{~N}$ was applied. A fungicide $\left(\mathrm{C}_{14} \mathrm{H}_{16} \mathrm{ClN}_{3} \mathrm{O}_{2}\right)$ was applied to the wheat plants to prevent powdery mildew. The shading experimental culture layout was shown in Figure 2.

\section{Experimental methods}

\section{Microclimate of the pot experiments}

A thermohygrometer provided by WS-1, Tianjin Fengyang Co. Ltd., China, was used to measure the relative humidity $(\mathrm{RH})$ and temperature $(\mathrm{T})$ of the shaded canopy. HOBO H21 automatic recording system of photosynthetically active radiation provided by Oneset, USA, was used to monitor photosynthetically active radiation (PAR). After the stem elongation stage $(\mathrm{BBCH} 30)$, all variables were monitored every $60 \mathrm{~min}$ between 8:00-18:00 on typical sunny days $24^{\text {th }}$ February, $16^{\text {th }}, 18^{\text {th }}, 28^{\text {th }}$ March, $1^{\text {st }}, 12^{\text {th }}$, $13^{\text {th }}, 24^{\text {th }}, 28^{\text {th }}$ April, $12^{\text {th }}, 13^{\text {th }}$ May, 2017 for each growing stage. Daily meteorological data, including $\mathrm{T}$, total radiation, and $\mathrm{RH}$, were recorded at a 5-min timestep using an automatic portable weather station (Watchdog 2900ET, Spectrum Technologies Inc., USA). 

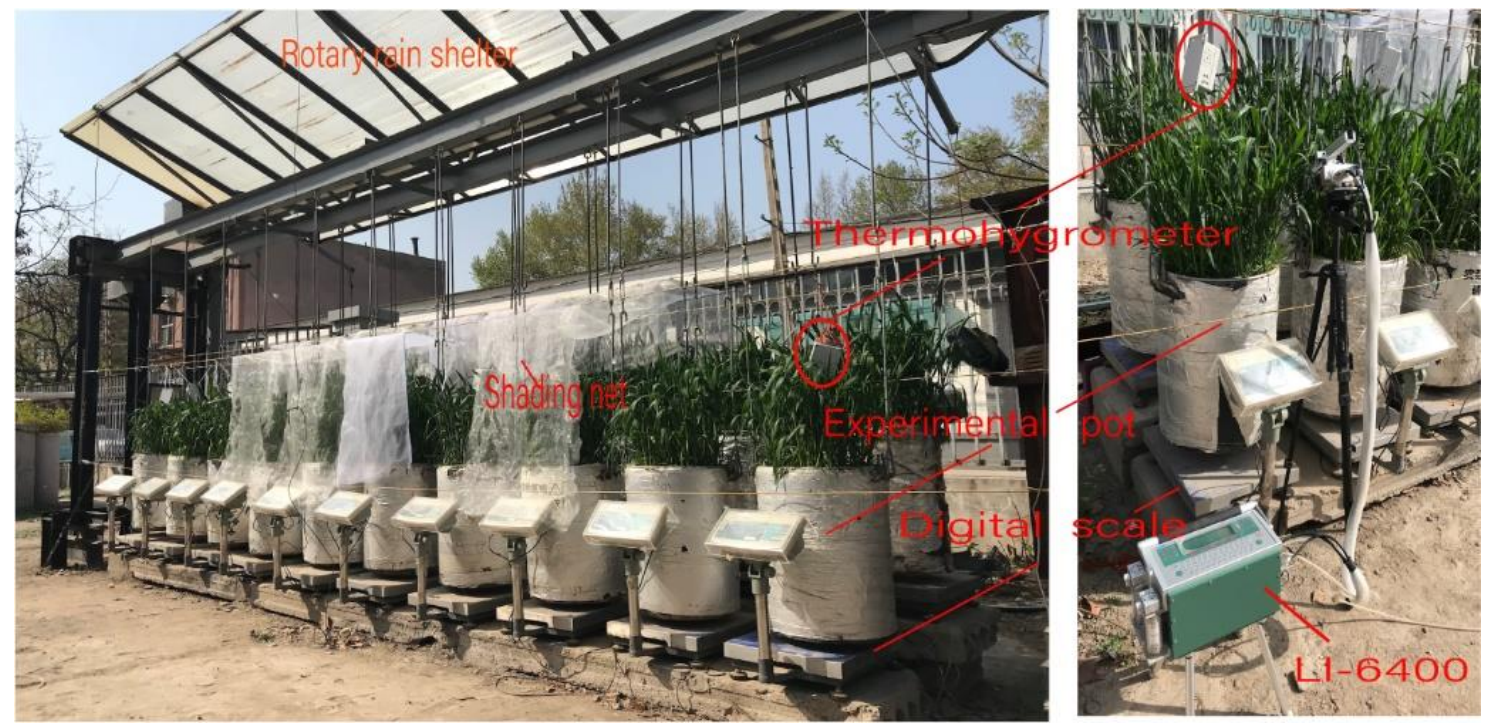

Figure 2. Image of shading experimental culture and equipment

\section{Leaf area index}

Five plants were randomly selected from each pot weekly after BBCH35. Leaf length (a) and greatest leaf width (b) were measured with a ruler, and the leaf area index (LAI) was calculated using the following equation:

$$
L A I=0.83 \times P \times \sum_{i}^{n}\left(\mathrm{a}_{i} \times b_{i}\right)
$$

where 0.83 is the compensation coefficient of wheat, $\mathrm{P}$ is the plant density of wheat, $\mathrm{n}$ is the number of wheat leaves per plant.

\section{Light response curves}

An open gas exchange system provides by LI-6400, LI-COR, Inc., Lincoln, Nebraska, USA, was used to measure the light response curves of fully expanded functional leaves of the CK plants. Illumination to the leaves was applied using a redblue LED light source (6400-02B). To activate the photosynthesis systems, the leaves were placed under the illumination of $1200 \mu \mathrm{mol} \mathrm{m} \mathrm{m}^{-2} \mathrm{~s}^{-1}$ for $20 \mathrm{~min}$. The air temperature was set to $25{ }^{\circ} \mathrm{C}$ and $\mathrm{CO}_{2}$ concentration was set at $400 \mathrm{~mol} \mathrm{~mol}^{-1}$. The photosynthetic light flux densities were set as 1700, 1500, 1200, 800, 600, 400, 200, $150,100,50$, and $0 \mu \mathrm{mol} \mathrm{m} \mathrm{m}^{-2}$. The measurements were implemented at 9:00-11:00 on sunny days, with three plants measured during each sample period. The nonrectangular hyperbola in the Farquhar model (Prioul and Chartier, 1977) was used to model the responses of net photosynthetic rate to different growth stages.

\section{Measurements of diurnal gas exchange}

The net photosynthetic rate $\left(\mathrm{P}_{\mathrm{n}}\right)$, stomatal conductance $\left(\mathrm{g}_{\mathrm{s}}\right)$, transpiration rate $(\mathrm{Tr})$, intrinsic WUE (WUE $\left.E_{\text {int }}=\mathrm{P}_{\mathrm{n}} / \mathrm{g}_{\mathrm{s}}\right)$, and instantaneous WUE $\left(\mathrm{WUE}_{\text {ins }}=\mathrm{P}_{\mathrm{n}} / \mathrm{Tr}\right.$ ) were estimated using the fully expanded functional leaves within the open gas exchange 
system (LI-6400; LI-COR, Inc., Lincoln, Nebraska USA). The experiment was run on sunny days from 8:00-18:00. Three plants from each treatment were measured every $2 \mathrm{~h}$, at four growth stages: stem elongation (BBCH 30-39), booting (BBCH 41-49), heading to flowering (BBCH 51-69), development of fruit (BBCH 70-79).

\section{Grain yield components}

Spike number per pot was enumerated and ten spikes per pot were selected to count the spike grain number. The yield and thousand-kernel weight were calculated after the drying of seeds.

\section{Evapotranspiration and WUE}

Water consumption was estimated by the daily weighing of the experimental pots at 8:00 through the use of digital scales provided by TCS-CC, Fuzhou Kedi Electronic Technology Co., Ltd., China. Temporal changes in water consumption were estimated through the use of a water balance equation incorporating the differences in the weights pots and their contents and the mass of added water among the different plants. ET was estimated as:

$$
E T=\frac{\Delta W+W_{a}}{s}
$$

where $\Delta \mathrm{W}$ is the difference in the pot weight $(\mathrm{kg}), \mathrm{W}_{\mathrm{a}}$ represents the mass of water added $(\mathrm{kg})$, and $\mathrm{S}$ represents the pot area $\left(\mathrm{m}^{2}\right)$. Weighing of the pots was conducted every $2 \mathrm{~h}$ on typical sunny days, namely on the $25^{\text {th }}$ March, $1^{\text {st }}$ April, $13^{\text {th }}$ April, and $28^{\text {th }}, 2017$ for all growing to estimate the typical daily ET.

The wheat water use efficiency (WUE) was calculated as:

$$
W U E=\frac{G Y}{\mathrm{ET}_{t}}
$$

where $G Y$ is grain yield of winter wheat $\left(\mathrm{kg} \mathrm{ha}^{-1}\right)$ and $\mathrm{ET}_{\mathrm{t}}$ is the total evapotranspiration over the wheat growing season $(\mathrm{mm})$.

\section{Statistical analysis}

A one-way analysis of variance (ANOVA) was conducted on all data. The significance of the differences between the treatments was determined through the Duncan's Range Test. All analysis was conducted in the SPSS statistical software (SPSS 20.0).

\section{Results}

\section{The effect of shading on LAI}

Figure 3 shows the LAI of each treatment from 2016 to 2017. No significant differences in LAI among treatments were observed during the same growth period. LAI first increased and then decreased with the growth of the wheat. The maximum LAI values of treatment $\mathrm{CK}$ and $\mathrm{S} 4$ occurred at $\mathrm{BBCH} 43$, while that of $\mathrm{S} 3, \mathrm{~S} 2$, and $\mathrm{S} 1$ was at BBCH49. S3 obtained the maximum LAI of 9.14, followed by S4, CK, S2, and 
S1. S4 obtained the maximum LAI between BBCH35 to BBCH43, whereas S3 obtained the maximum LAI during the subsequent growth stage. The decrease in LAI within CK accelerated after BBCH49.

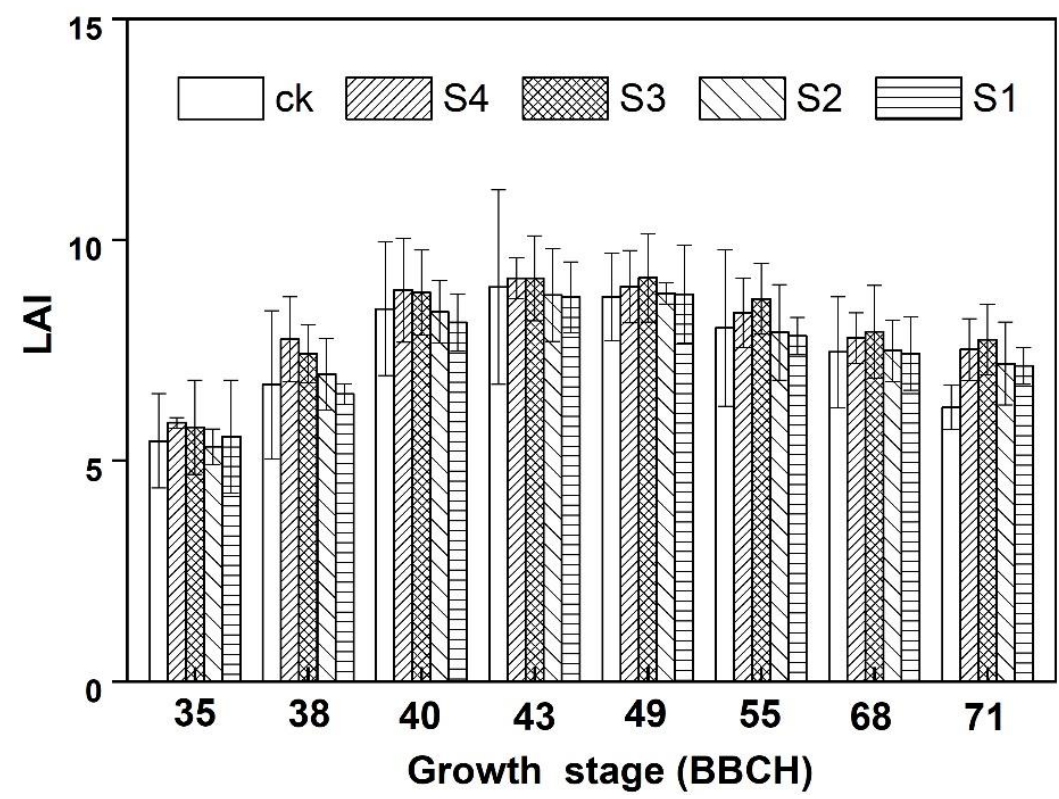

Figure 3. Leaf area index from $B B C H 35$ to 71 within a shading experiment using the winter wheat. CK refers to the "no shading" treatment (control), whereas $S 4, S 3$, S2, and S1 refer to light saturation point $(L S P)+400 \mu \mathrm{mol} \mathrm{m} \mathrm{m}^{-2} \mathrm{~s}^{-1}, \mathrm{LSP}+100 \mu \mathrm{mol} \mathrm{m} \mathrm{s}^{-2}, \mathrm{LSP}-$ $200 \mu \mathrm{mol} \mathrm{m} \mathrm{m}^{-1}$, and LSP $-500 \mu \mathrm{mol} \mathrm{m} \mathrm{s}^{-2}$, respectively. Vertical bars indicate standard error. The same as below

\section{Shading effect on changes to $R H, T$, and PAR in the wheat canopy}

The design of the shading experiment ensured a significant difference between PAR and other treatments after the shading intensity was reached. S1 reached the shading light intensity at 8:00 under a greater degree of shading, whereas S4 reached the shading light intensity at 11:00. The maximum PAR occurred at 12:00 during which there was significant difference between the treatments. Compared to CK, the PAR at S4, S3, S2, and S1 decreased by $14.7 \%, 29.3 \%, 39.5 \%$, and $57.6 \%$ with increased shading, respectively. A maximum canopy temperature of $31.33{ }^{\circ} \mathrm{C}$ was recorded at $14: 00$. The temperatures of S4, S3, S2, and S1 decreased by $1.4 \%, 4.3 \%, 8.0 \%$, and $10.3 \%$ with increased shading, respectively. Significant differences in T were evident between CK and S4 and between S2 and S4. The RH value gradually increased with increasing shading during the shading period (Table 2).

\section{The effect of shading on photosynthetic indices of functional leaves}

\section{Shading effect on the Pn}

As shown in Figure 4, $\mathrm{P}_{\mathrm{n}}$ generally decreased with increasing shading during the four growth stages. Since BBCH55 (Fig. 4B), the $\mathrm{P}_{\mathrm{n}}$ of S4 started to increase during individual periods, namely, BBCH68 at 12:00-14:00, BBCH68 at 10:00-14:00 (Fig. 4C), and BBCH75 at 10:00-12:00 (Fig. 4D). although there was no significant 
difference with the $\mathrm{CK}$ treatment. The changes in $\mathrm{P}_{\mathrm{n}}$ at $\mathrm{BBCH} 47$ and $\mathrm{BBCH} 75$ were similar among CK, S4, and S3 (Fig. 4A, C, D) over the four growth stages during which $P_{n}$ first increased, reaching a maximum at 10:00-12:00, following which it gradually decreased. In contrast, the $\mathrm{P}_{\mathrm{n}}$ of BBCH55 (Fig. 4B) gradually decreased. The $\mathrm{P}_{\mathrm{n}}$ values of $\mathrm{CK}$ and $\mathrm{S} 4$ were significantly reduced during the "midday depression" phenomenon in BBCH47 and BBCH55 at 12:00-14:00 (Fig. 4A, B). Besides, the significance of differences in $\mathrm{P}_{\mathrm{n}}$ and the period of significant difference between treatments under shading gradually decreased as wheat matured. The $\mathrm{P}_{\mathrm{n}}$ in $\mathrm{BBCH} 75$ showed a significant difference only from 12:00-14:00, with no significant differences between adjacent treatments (Fig. 4D).

Table 2. Relative humidity $(R H)$, temperature $(T)$, and photosynthetically active radiation $(P A R)$ under each treatment

\begin{tabular}{|c|c|c|c|c|c|c|c|c|c|c|c|}
\hline Treatment & $8: 00$ & 9:00 & 10:00 & 11:00 & 12:00 & 13:00 & $14: 00$ & $15: 00$ & $16: 00$ & $17: 00$ & 18:00 \\
\hline & \multicolumn{11}{|c|}{ PAR $\left(\mu \mathrm{mol} \mathrm{m} \mathrm{m}^{-2} \mathrm{~s}^{-1}\right)$} \\
\hline $\mathrm{CK}$ & $\begin{array}{c}1206 \pm \\
60.19 \mathrm{a} \dagger\end{array}$ & $\begin{array}{l}1501 \pm \\
16.17 \mathrm{a}\end{array}$ & $\begin{array}{c}1661 \pm \\
9.29 \mathrm{a}\end{array}$ & $\begin{array}{l}1846 \pm \\
30.02 \mathrm{a}\end{array}$ & $\begin{array}{l}1950 \pm \\
40.34 \mathrm{a}\end{array}$ & $\begin{array}{l}1942 \pm \\
52.84 \mathrm{a}\end{array}$ & $\begin{array}{l}1940 \pm \\
13.86 \mathrm{a}\end{array}$ & $\begin{array}{l}1909 \pm \\
15.01 \mathrm{a}\end{array}$ & $\begin{array}{c}1703.67 \pm \\
14.74 \mathrm{a}\end{array}$ & $\begin{array}{l}1218 \pm \\
2.52 \mathrm{a}\end{array}$ & $\begin{array}{c}113 \pm \\
1.53\end{array}$ \\
\hline S4 & $\begin{array}{l}1192 \pm \\
16.26 \mathrm{a}\end{array}$ & $\begin{array}{c}1503 \pm \\
5.03 \mathrm{a}\end{array}$ & $\begin{array}{l}1669 \pm \\
10.02 \mathrm{a}\end{array}$ & $\frac{1597 \pm}{9.45 b}$ & $\underline{1663 \pm}$ & $\underline{\underline{1621 \pm}} \underline{\underline{51.16 \mathrm{~b}}}$ & $\underline{\underline{1660 \pm}}$ & $\underline{1473 \pm}$ & $\underline{\underline{1461 \pm}}$ & $\begin{array}{l}1225 \pm \\
17.62 \mathrm{a}\end{array}$ & $\begin{array}{c}113 \pm \\
1.53\end{array}$ \\
\hline S3 & $\begin{array}{c}1186 \pm \\
6.66 \mathrm{a}\end{array}$ & $\begin{array}{c}1503 \pm \\
4.58 \mathrm{a}\end{array}$ & $\begin{array}{l}\underline{1346 \pm} \\
\underline{28.15 b}\end{array}$ & $\begin{array}{l}\underline{1231 \pm} \\
\underline{14.42 \mathrm{c}}\end{array}$ & $\begin{array}{l}\underline{1379 \pm} \\
\underline{41.51 \mathrm{c}}\end{array}$ & $\begin{array}{l}\underline{1445 \pm} \\
\underline{17.35 \mathrm{c}}\end{array}$ & $\begin{array}{l}\underline{1367 \pm} \\
\underline{16.52 \mathrm{c}}\end{array}$ & $\underline{1123 \pm}$ & $\begin{array}{l}\underline{1118 \pm} \\
\underline{11.85 \mathrm{c}}\end{array}$ & $\begin{array}{l}1204 \pm \\
13.43 \mathrm{a}\end{array}$ & $\begin{array}{c}114 \pm \\
0.58\end{array}$ \\
\hline S2 & $\begin{array}{l}1155 \pm \\
31.01 \mathrm{a}\end{array}$ & $\underline{\underline{875 \pm}}$ & $\begin{array}{c}\underline{794 \pm} \\
\underline{18.25 \mathrm{c}}\end{array}$ & $\underline{\underline{981 \pm}} \underline{27.15 \mathrm{~d}}$ & $\underline{\underline{1179 \pm}}$ & $\underline{\underline{1137 \pm}}$ & $\underline{\underline{1108 \pm}}$ & $\underline{\frac{889 \pm}{2.65 \mathrm{~d}}}$ & $\underline{\underline{840 \pm}} \underline{\underline{25.16 \mathrm{~d}}}$ & $\underline{\underline{1161 \pm}} \underline{15.53 \mathrm{~b}}$ & $\begin{array}{c}114 \pm \\
0.58\end{array}$ \\
\hline S1 & $\underline{552 \pm}$ & $\frac{585 \pm}{\underline{6.08 \mathrm{c}}}$ & $\frac{676 \pm}{8.62 \mathrm{~d}}$ & $\underline{632 \pm}$ & $\underline{\underline{826 \pm}} \underline{\underline{63.11 \mathrm{e}}}$ & $\frac{807.67 \pm}{\underline{3.21 \mathrm{e}}}$ & $\underline{795 \pm}$ & $\frac{715 \pm}{1.00 \mathrm{e}}$ & $\frac{663 \pm}{17.09 \mathrm{e}}$ & $\underline{\underline{567 \pm}} \underline{\underline{21.03 \mathrm{c}}}$ & $\begin{array}{c}113 \pm \\
1.00\end{array}$ \\
\hline \multirow[t]{2}{*}{$\mathrm{p}$} & $0 * * *$ & $0 * * *$ & $0 * * *$ & $0 * * *$ & $0 * * *$ & $0 * * *$ & $0 * * *$ & $0 * * *$ & $0 * * *$ & $0 * * *$ & 0.941 \\
\hline & \multicolumn{11}{|c|}{$\mathrm{T}\left({ }^{\circ} \mathrm{C}\right)$} \\
\hline CK & $\begin{array}{c}14.97 \pm \\
0.45\end{array}$ & $\begin{array}{c}20.87 \pm \\
0.91\end{array}$ & $\begin{array}{c}24.47 \pm \\
0.51 \mathrm{a}\end{array}$ & $\begin{array}{c}26.23 \pm \\
0.25 \mathrm{a}\end{array}$ & $\begin{array}{c}28.03 \pm \\
0.35 \mathrm{a}\end{array}$ & $\begin{array}{c}30.17 \pm \\
0.70 \mathrm{a}\end{array}$ & $\begin{array}{c}31.33 \pm \\
0.29 \mathrm{a}\end{array}$ & $\begin{array}{c}30.93 \pm \\
0.60\end{array}$ & $\begin{array}{c}29.53 \pm \\
0.45\end{array}$ & $\begin{array}{c}27.77 \pm \\
0.15\end{array}$ & $\begin{array}{c}26.17 \pm \\
0.06\end{array}$ \\
\hline S4 & $\begin{array}{c}15.03 \pm \\
0.21\end{array}$ & $\begin{array}{c}21.00 \pm \\
1.31\end{array}$ & $\begin{array}{c}23.33 \pm \\
0.12 b\end{array}$ & $\underline{\underline{25.47 \pm}} \underline{\underline{0.61 \mathrm{ab}}}$ & $\underline{\underline{27.30 \pm}} \underline{\underline{0.56 \mathrm{~b}}}$ & $\begin{array}{l}\underline{29.40 \pm} \\
\underline{1.06 \mathrm{ab}}\end{array}$ & $\begin{array}{l}\underline{30.90 \pm} \\
\underline{0.95 \mathrm{ab}}\end{array}$ & $\underline{\underline{31.00 \pm}} \underline{\underline{2.35}}$ & $\frac{29.30 \pm}{\underline{1.57}}$ & $\begin{array}{c}28.03 \pm \\
0.32\end{array}$ & $\begin{array}{c}26.10 \pm \\
0.00\end{array}$ \\
\hline S3 & $\begin{array}{c}14.97 \pm \\
0.32\end{array}$ & $\begin{array}{c}20.50 \pm \\
0.50\end{array}$ & $\begin{array}{l}\underline{22.87 \pm} \\
\underline{0.12 \mathrm{bc}}\end{array}$ & $\begin{array}{l}\underline{25.10 \pm} \\
\underline{0.26 \mathrm{bc}}\end{array}$ & $\frac{26.57 \pm}{\underline{0.49 \mathrm{c}}}$ & $\begin{array}{l}\underline{28.83 \pm} \\
\underline{0.21 \mathrm{bc}}\end{array}$ & $\underline{\underline{29.97 \pm}} \underline{\underline{0.50 \mathrm{~b}}}$ & $\frac{29.97 \pm}{\underline{0.40}}$ & $\frac{29.60 \pm}{\underline{0.20}}$ & $\begin{array}{c}27.83 \pm \\
0.21\end{array}$ & $\begin{array}{c}26.10 \pm \\
0.10\end{array}$ \\
\hline S2 & $\begin{array}{c}15.20 \pm \\
0.10\end{array}$ & $\frac{20.40 \pm}{\underline{2.21}}$ & $\begin{array}{l}\underline{22.50 \pm} \\
\underline{0.44 \mathrm{~cd}}\end{array}$ & $\underline{\underline{24.57 \pm}} \underline{\underline{0.81 \mathrm{bc}}}$ & $\underline{\underline{25.83 \pm}} \underline{\underline{0.21 \mathrm{~d}}}$ & $\begin{array}{l}\underline{27.97 \pm} \\
\underline{0.23 \mathrm{~cd}}\end{array}$ & $\underline{\underline{28.80 \pm}} \underline{\underline{0.17 \mathrm{c}}}$ & $\frac{28.87 \pm}{\underline{1.01}}$ & $\frac{28.53 \pm}{\underline{0.64}}$ & $\frac{27.83 \pm}{\underline{0.55}}$ & $\begin{array}{c}26.07 \pm \\
0.06\end{array}$ \\
\hline S1 & $\frac{15.37 \pm}{\underline{0.31}}$ & $\frac{19.37 \pm}{\underline{0.40}}$ & $\frac{22.17 \pm}{\underline{0.38 \mathrm{~d}}}$ & $\frac{24.13 \pm}{\underline{0.59 \mathrm{c}}}$ & $\underline{\underline{25.17 \pm}} \underline{\underline{0.15 \mathrm{~d}}}$ & $\frac{27.37 \pm}{\underline{0.32 \mathrm{~d}}}$ & $\frac{28.10 \pm}{\underline{0.46 \mathrm{c}}}$ & $\frac{28.27 \pm}{\underline{0.15}}$ & $\frac{28.27 \pm}{\underline{0.31}}$ & $\frac{27.43 \pm}{\underline{0.42}}$ & $\begin{array}{c}26.20 \pm \\
0.10\end{array}$ \\
\hline \multirow[t]{2}{*}{$\mathrm{p}$} & 0.452 & 0.556 & $0 * * *$ & $0.007 * *$ & $0 * * *$ & $0.001 * *$ & $0 * * *$ & 0.064 & 0.221 & 0.407 & 0.229 \\
\hline & \multicolumn{11}{|c|}{ RH (\%) } \\
\hline CK & $\begin{array}{c}68.33 \pm \\
2.52\end{array}$ & $\begin{array}{c}55.00 \pm \\
2.65\end{array}$ & $\begin{array}{c}47.00 \pm \\
1.00 \mathrm{c}\end{array}$ & $\begin{array}{c}39.00 \pm \\
1.00 \mathrm{e}\end{array}$ & $\begin{array}{c}33.67 \pm \\
0.80 \mathrm{~d}\end{array}$ & $\begin{array}{c}27.37 \pm \\
0.55 \mathrm{~d}\end{array}$ & $\begin{array}{c}26.87 \pm \\
0.81 \mathrm{e}\end{array}$ & $\begin{array}{c}25.00 \pm \\
1.00 \mathrm{~d}\end{array}$ & $\begin{array}{c}23.00 \pm \\
2.65\end{array}$ & $\begin{array}{c}22.33 \pm \\
2.52\end{array}$ & $\begin{array}{c}25.67 \pm \\
2.52\end{array}$ \\
\hline S4 & $\begin{array}{c}69.67 \pm \\
1.53\end{array}$ & $\begin{array}{c}57.00 \pm \\
2.00\end{array}$ & $\begin{array}{c}50.00 \pm \\
1.00 \mathrm{~b}\end{array}$ & $\frac{41.33 \pm}{\underline{0.58 \mathrm{~d}}}$ & $\frac{35.67 \pm}{\underline{0.76 \mathrm{c}}}$ & $\frac{30.33 \pm}{\underline{2.08 \mathrm{c}}}$ & $\begin{array}{l}\underline{28.33 \pm} \\
\underline{0.58 \mathrm{~d}}\end{array}$ & $\underline{26.00 \pm}$ & $\frac{24.00 \pm}{\underline{2.65}}$ & $\begin{array}{c}25.00 \pm \\
1.00\end{array}$ & $\begin{array}{c}27.67 \pm \\
1.15\end{array}$ \\
\hline S3 & $\begin{array}{c}70.67 \pm \\
1.15\end{array}$ & $\begin{array}{c}57.67 \pm \\
2.52\end{array}$ & $\frac{51.67 \pm}{1.53 \mathrm{ab}}$ & $\frac{43.13 \pm}{\underline{0.32 \mathrm{c}}}$ & $\underline{\underline{36.53 \pm}} \underline{\underline{0.92 \mathrm{c}}}$ & $\underline{\underline{31.27 \pm}} \underline{\underline{1.07 \mathrm{bc}}}$ & $\frac{29.83 \pm}{\underline{0.76 \mathrm{c}}}$ & $\underline{\underline{27.67 \pm}}$ & $\frac{24.00 \pm}{\underline{2.00}}$ & $\begin{array}{c}26.33 \pm \\
1.53\end{array}$ & $\begin{array}{c}28.00 \pm \\
1.00\end{array}$ \\
\hline S2 & $\begin{array}{c}69.00 \pm \\
1.00\end{array}$ & $\frac{58.33 \pm}{\underline{3.06}}$ & $\frac{52.17 \pm}{\underline{0.38 \mathrm{a}}}$ & $\frac{45.37 \pm}{\underline{0.32 b}}$ & $\underline{\underline{38.83 \pm}} \underline{\underline{0.80 \mathrm{~b}}}$ & $\underline{\underline{33.33 \pm}} \underline{\underline{2.08 \mathrm{ab}}}$ & $\frac{32.13 \pm}{\underline{0.32 \mathrm{~b}}}$ & $\underline{\underline{28.67 \pm}} \underline{1.53 \mathrm{ab}}$ & $\frac{25.33 \pm}{\underline{2.08}}$ & $\frac{\underline{26.00 \pm}}{\underline{5.29}}$ & $\begin{array}{c}28.67 \pm \\
1.53\end{array}$ \\
\hline S1 & $\frac{69.67 \pm}{\underline{1.53}}$ & $\frac{59.07 \pm}{\underline{1.10}}$ & $\begin{array}{l}\underline{52.50 \pm} \\
\underline{1.05 \mathrm{a}}\end{array}$ & $\frac{47.63 \pm}{\underline{0.65 \mathrm{a}}}$ & $\frac{40.23 \pm}{\underline{0.32 \mathrm{a}}}$ & $\begin{array}{l}\underline{35.67 \pm} \\
\underline{1.53 \mathrm{a}}\end{array}$ & $\frac{33.30 \pm}{\underline{0.61 \mathrm{a}}}$ & $\frac{30.67 \pm}{\underline{1.53 \mathrm{a}}}$ & $\frac{28.33 \pm}{\underline{0.58}}$ & $\frac{29.33 \pm}{\underline{1.53}}$ & $\begin{array}{c}29.00 \pm \\
1.00\end{array}$ \\
\hline $\mathrm{p}$ & 0.525 & 0.34 & $0 * * *$ & $0 * * *$ & $0 * * *$ & $0.001 * *$ & $0 * * *$ & $0.003 * *$ & 0.082 & 0.121 & 0.152 \\
\hline
\end{tabular}

$\uparrow$ Data followed by the same letter in a column represent nonsignificant differences among the treatments as determined through the least significant difference (LSD) test $(\mathrm{P}=0.05)$. The underlined number is the value under shading time. CK represents the "no shading" treatment (control), whereas S4, S3, S2, and S1 refer to the light saturation point (LSP) $+400 \mu \mathrm{mol} \mathrm{m}{ }^{-2} \mathrm{~s}^{-1}, \mathrm{LSP}$ $+100 \mu \mathrm{mol} \mathrm{m} \mathrm{s}^{-2}$, LSP $-200 \mu \mathrm{mol} \mathrm{m}{ }^{-2} \mathrm{~s}^{-1}$, and LSP $-500 \mu \mathrm{mol} \mathrm{m} \mathrm{m}^{-2}$, respectively. Data P-P value of shading treatment. *, significance at the .05 level; **, significance at the .01 level; ***, significance at the .001 level. Values are means \pm standard deviation of four independent replicates 


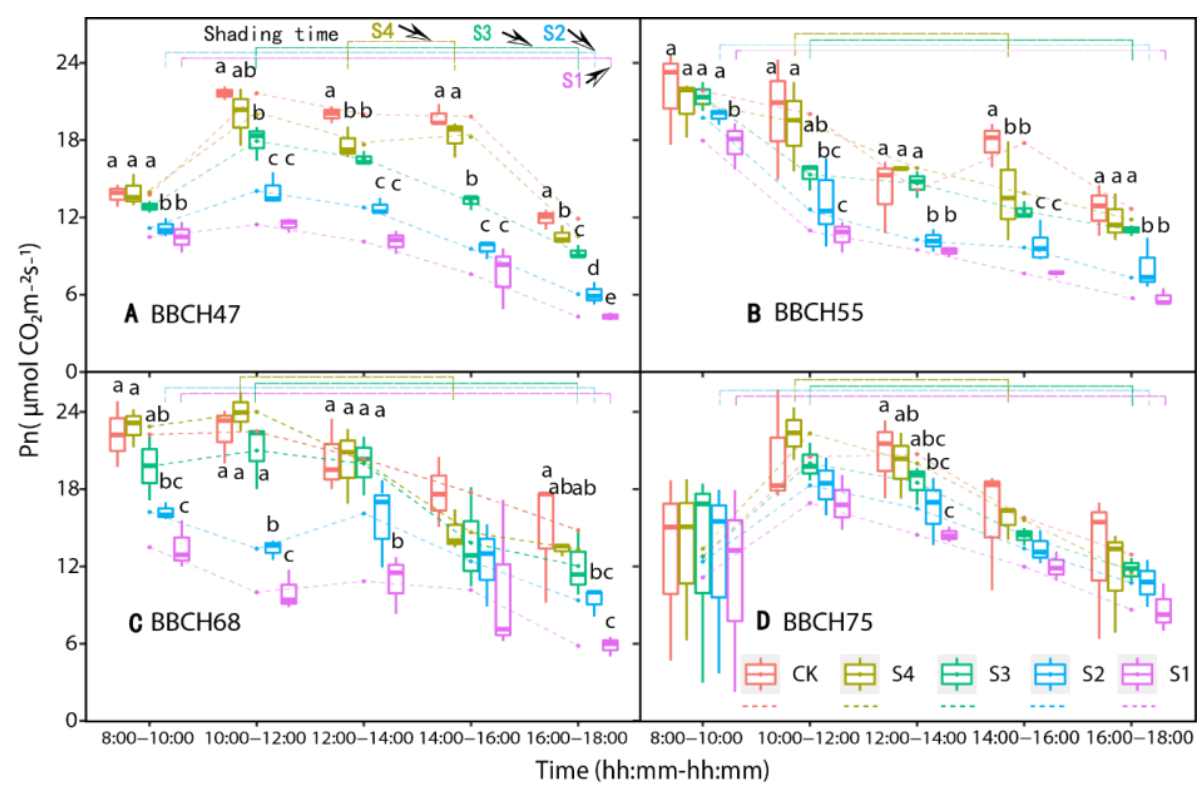

Figure 4. The effect of shading on the net photosynthetic rate of winter wheat functional leaves in 2017. The error bars represent standard deviations of the averages of the four replicates. Different letters for each trait represent a significant difference according to a one-way analysis of variance (ANOVA) after testing using the least significant difference (LSD) multiple comparison $(P<0.05)$. CK refers to the "no shading" treatment (control), whereas $S 4, S 3, S 2$, and S1 refer to light saturation point $(L S P)+400 \mu \mathrm{mol} \mathrm{m} \mathrm{m}^{-2} \mathrm{~s}^{-1} L S P+100 \mu \mathrm{mol} \mathrm{m} \mathrm{m}^{-2} \mathrm{~s}^{-1}, L S P-$ $200 \mu \mathrm{mol} \mathrm{m} \mathrm{m}^{-1}$, and $L S P-500 \mu \mathrm{mol} \mathrm{m} \mathrm{s}^{-2}$, respectively. The same as below

\section{Effect of shading on stomatal conductance}

As shown in Figure 5, there was generally a decreasing trend in stomatal conductance $\left(\mathrm{g}_{\mathrm{s}}\right)$ with increasing shading intensity.

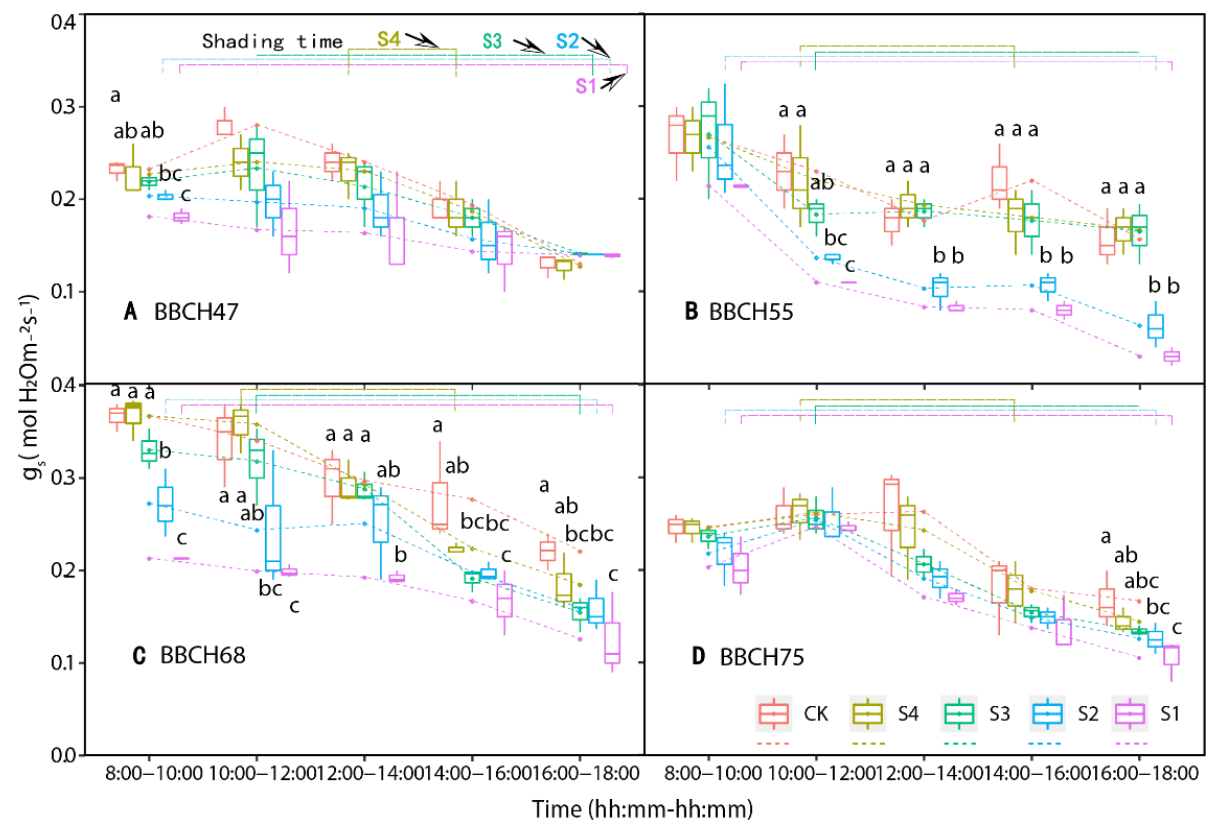

Figure 5. The impact of shading on the stomatal conductance of winter wheat functional leaves within a shading experiment in 2017 


\section{The effect of shading on the diurnal transpiration rate}

The diurnal transpiration rate (Tr) first increased and then decreased, with the maximum values occurring mainly during 12:00-14:00 at BBCH55 and BBCH75 (Fig. 6B, D). The maximum Tr values of CK and S4 over BBCH47 occurred at 14:0016:00, whereas those of the remaining treatments were at 12:00-14:00 (Fig. 6A). Only $\mathrm{CK}$ achieved a maximum value of $9.03 \mathrm{~mol} \mathrm{H}_{2} \mathrm{O} \mathrm{m}^{-2} \mathrm{~s}^{-1}$ over BBCH68 at 14:00-16:00, whereas those of the remaining treatments were at 12:00-14:00 (Fig. 6C). Shading had a significant effect on leaf $\operatorname{Tr}$ at all growth stages, with $\operatorname{Tr}$ decreasing gradually with increasing shading in all treatments, except $\mathrm{S} 4$ at BBCH55 and BBCH75 (Fig. 6B, D). The degree of significant difference between treatments and the period decreased with increasing wheat maturity. The $\mathrm{Tr}$ values of $\mathrm{S} 4, \mathrm{~S} 3, \mathrm{~S} 2$, and $\mathrm{S} 1$ were reduced by $4.8 \%$, $11.4 \%, 22.3 \%$, and $32.8 \%$, in $\mathrm{BBCH} 47,0.8 \%, 7.3 \%, 29.5 \%$, and $44.3 \%$ in $\mathrm{BBCH} 55$, $2.3 \%, 6.3 \%, 16.5 \%$, and $20.7 \%$ in $\mathrm{BBCH} 68$, and $-1.6 \%, 3.0 \%, 9.6 \%$, and $16.4 \%$ in $\mathrm{BBCH} 75$, respectively.

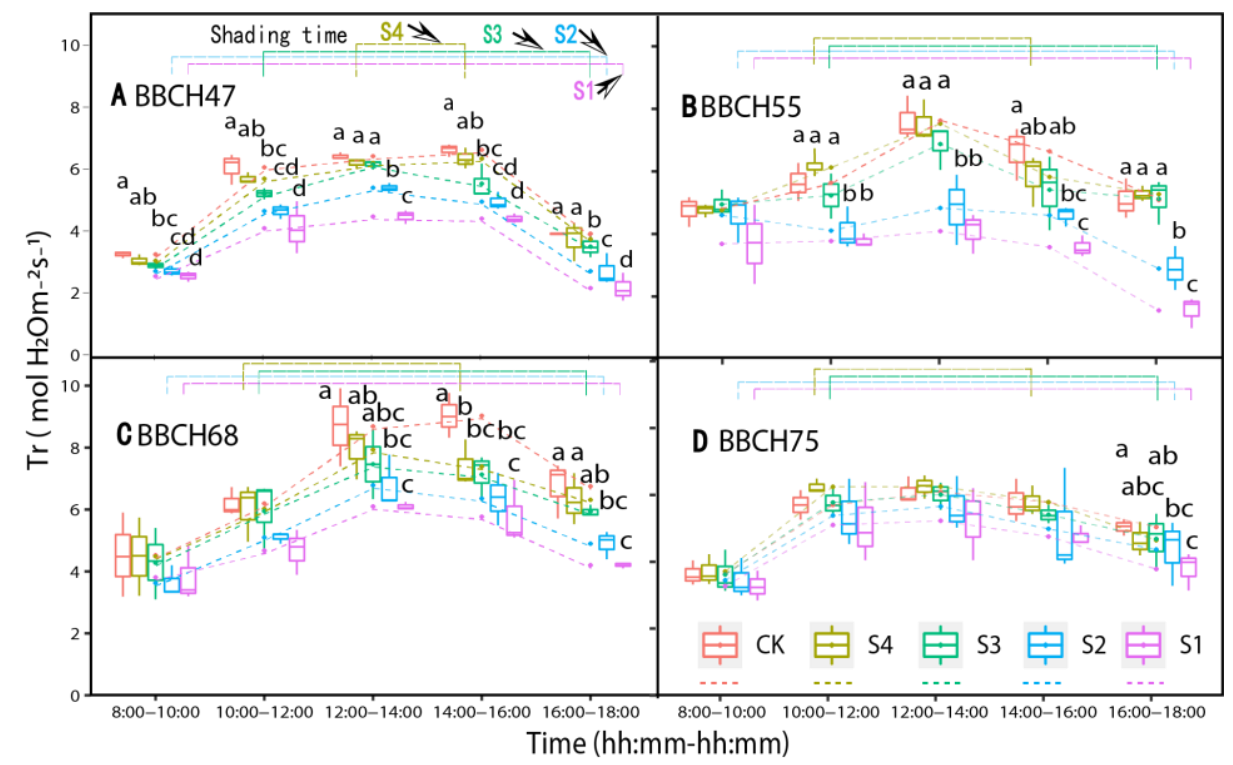

Figure 6. The impact of shading on the transpiration of winter wheat functional leaves within a shading experiment in 2017

\section{Diurnal variations in winter wheat water consumption of under shading}

Daytime hourly evapotranspiration (ET) in 2017 showed a bimodal variation at BBCH47, BBCH55, and BBCH68 (Fig. 7A, B, C), reaching a maximum at 12:00-13:00 and another peak at 14:00-15:00, with a trough between. ET at BBCH75 first increased and then decreased, with the maximum at 13:00-14:00 (Fig. 7D). The maximum hourly ET of $2.18 \mathrm{~mm}$ was observed at 12:00-13:00 during BBCH68 of S4, whereas the ET of the other treatments during the four growth stages gradually decreased with increasing shading. Shading showed periods of significant effect on ET during each growth stage, with the most and least effect at $\mathrm{BBCH} 68$ and $\mathrm{BBCH} 75$, respectively. There were no significant differences between adjacent treatments, except for a few periods. The average water consumption (ET $\mathrm{ET}_{\text {day }}$ ) values of $\mathrm{S} 1, \mathrm{~S} 2, \mathrm{~S} 3$, and $\mathrm{S} 4$, during the four growth periods at 8:00-18:00 were $7.83,9.76,13.4$, and $11.86 \mathrm{~mm}$, respectively. The maximum 
$\mathrm{ET}_{\text {day }}$ of $15.25 \mathrm{~mm}$ was observed during BBCH68 for S4. Shading had a significant effect on $\mathrm{ET}_{\text {day }}$ among all growth periods, except for BBCH55. ET day gradually decreased under S4, S3, S2 and S1 by 1.6, 6.7, 23.4, and 30.5\% at BBCH47, -1.3, 10.4, 13.3, and $16.4 \%$ at BBCH55, $-1.9,1.4,17.0$, and $26.8 \%$ at BBCH68, and -5.3, 8.2, 16.3 , and $16.7 \%$ at $\mathrm{BBCH} 75$, respectively.

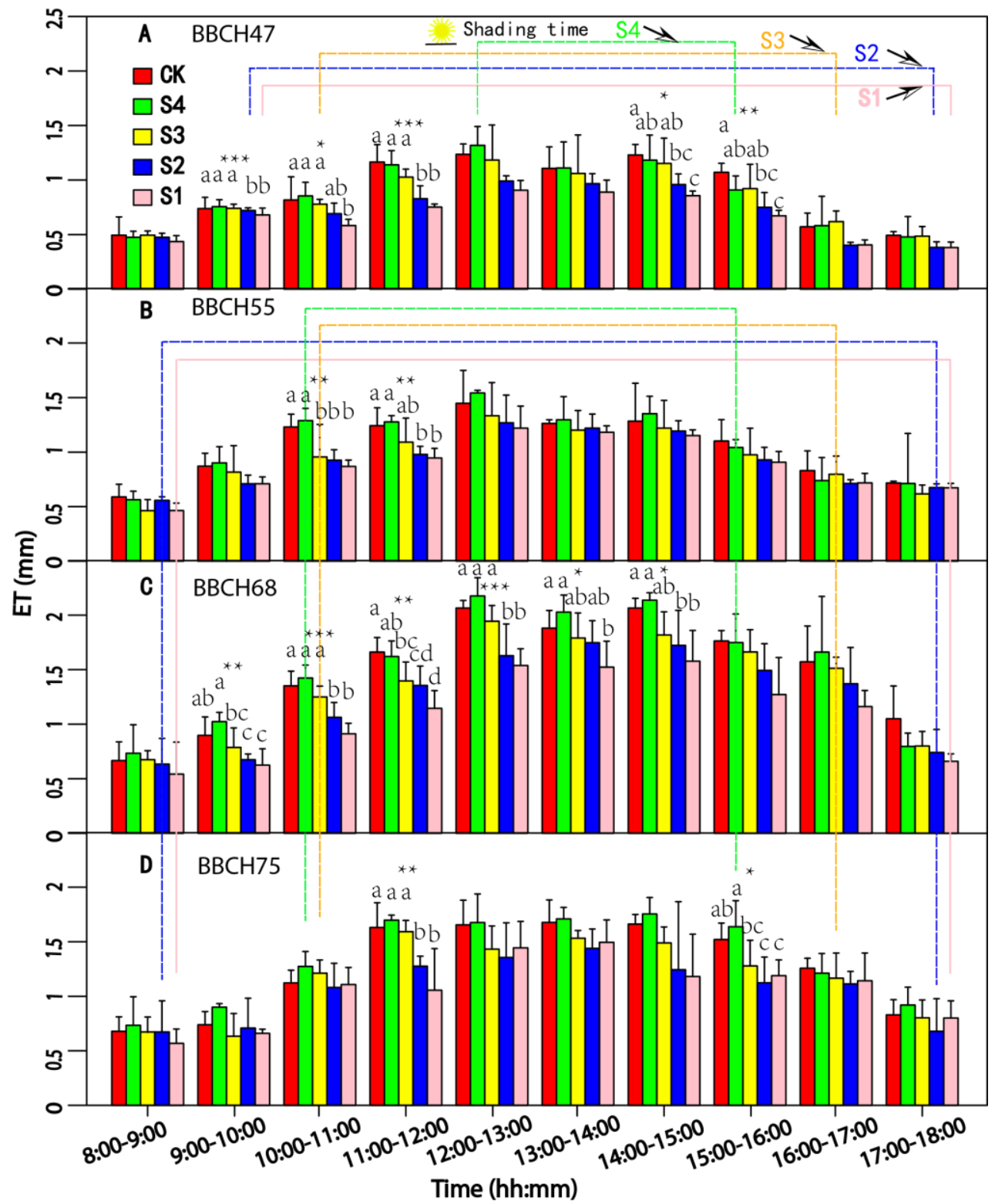

Figure 7. Evapotranspiration of the winter wheat at 8:00-18:00 during sunny days during different growing stages in 2017

\section{The effect of shading on water use efficiency of functional leaves}

\section{Shading effect on the $W U E_{\text {ins }}$ of functional leaves}

The diurnal variations in WUEins gradually decreased among all growth stages (Table 3). Besides for BBCH75, WUEins decreased rapidly at 8:00-14:00, with significant differences between periods, following which WUEins stabilized at 14:00- 
18:00. The difference in WUEins gradually increased with increasing shading. WUEins decreased gradually in each treatment for $\mathrm{BBCH} 55$, whereas the rate of increase in $\mathrm{CK}$ accelerated. The rate of decrease in WUEins tended to stabilize as shading increased, with the difference in WUEins between each time period not significant.

Table 3. The effect of shading on instantaneous water use efficiency in 2017 ( $\mu \mathrm{mol} \mathrm{CO} \mathrm{CO}_{2}$ $\mathrm{mol}^{-1} \mathrm{H}_{2} \mathrm{O}$ )

\begin{tabular}{|c|c|c|c|c|c|c|}
\hline Growth stage & Treatment & 8:00:00-10:00 & 10:00-12:00 & 12:00-14:00 & 14:00-16:00 & 16:00-18:00 \\
\hline \multirow{6}{*}{ BBCH47 } & CK & $4.29 \pm 0.13 \mathrm{bc} A \dagger$ & $3.59 \pm 0.23 \mathrm{a} \mathrm{B}$ & $3.12 \pm 0.07$ a C & $3.00 \pm 0.09 \mathrm{a} \mathrm{C}$ & $3.05 \pm 0.18 \mathrm{a} \mathrm{C}$ \\
\hline & S4 & $4.40 \pm 0.14 \mathrm{a} \mathrm{A}$ & $3.51 \pm 0.31 \mathrm{ab} B$ & $2.85 \pm 0.14 \mathrm{~b} \mathrm{C}$ & $2.88 \pm 0.11 \mathrm{aC}$ & $2.86 \pm 0.38$ a C \\
\hline & S3 & $4.36 \pm 0.03 \mathrm{ab} \mathrm{A}$ & $\underline{3.44 \pm 0.15 \mathrm{ab} \mathrm{B}}$ & $2.69 \pm 0.07 \mathrm{~b} \mathrm{C}$ & $2.41 \pm 0.20 \mathrm{~b} \mathrm{D}$ & $2.67 \pm 0.16 \mathrm{ab} \mathrm{C}$ \\
\hline & S2 & $\underline{4.14 \pm 0.02 \mathrm{c} \mathrm{A}}$ & $\underline{3.03 \pm 0.19 \mathrm{bc} \mathrm{B}}$ & $2.37 \pm 0.06 \mathrm{c} \mathrm{C}$ & $\underline{1.94 \pm 0.10 \mathrm{cD}}$ & $2.25 \pm 0.13 \mathrm{bc} \mathrm{C}$ \\
\hline & S1 & $\underline{4.12 \pm 0.21 \mathrm{c} \mathrm{A}}$ & $\underline{2.86 \pm 0.45 \mathrm{c} \mathrm{B}}$ & $\underline{2.27 \pm 0.09 \mathrm{c} \mathrm{BC}}$ & $\underline{1.72 \pm 0.50 \mathrm{c} \mathrm{C}}$ & $\underline{2.04 \pm 0.27 \mathrm{c} \mathrm{C}}$ \\
\hline & $\mathrm{P}$ & $0.004 * *$ & $0.04 *$ & $0 * * *$ & $0 * * *$ & $0.002 * *$ \\
\hline \multirow{6}{*}{ BBCH55 } & $\mathrm{CK}$ & $4.60 \pm 0.34 \mathrm{~A}$ & $3.54 \pm 0.50 \mathrm{a} \mathrm{B}$ & $1.85 \pm 0.29$ a D & $2.70 \pm 0.09$ a C & $2.51 \pm 0.13 \mathrm{a} \mathrm{C}$ \\
\hline & S4 & $4.59 \pm 0.29 \mathrm{~A}$ & $\underline{3.15 \pm 0.47 \mathrm{ab} \mathrm{B}}$ & $\underline{2.12 \pm 0.17 \mathrm{a} \mathrm{C}}$ & $2.38 \pm 0.37 \mathrm{a} \mathrm{C}$ & $2.25 \pm 0.24 \mathrm{a} \mathrm{C}$ \\
\hline & S3 & $4.56 \pm 0.16 \mathrm{~A}$ & $2.97 \pm 0.23 \mathrm{ab} \mathrm{B}$ & $2.15 \pm 0.10 \mathrm{aC}$ & $2.39 \pm 0.49 \mathrm{aC}$ & $2.19 \pm 0.25 \mathrm{a} \mathrm{C}$ \\
\hline & S2 & $\underline{4.44 \pm 0.68 \mathrm{~A}}$ & $2.43 \pm 0.50 \mathrm{bc} \mathrm{B}$ & $\underline{1.46 \pm 0.18 \mathrm{~b} \mathrm{C}}$ & $\underline{1.35 \pm 0.30 \mathrm{~b} \mathrm{C}}$ & $\underline{1.01 \pm 0.42 \mathrm{~b} \mathrm{C}}$ \\
\hline & S1 & $\underline{4.44 \pm 1.10 \mathrm{~A}}$ & $1.85 \pm 0.25 \mathrm{c} \mathrm{B}$ & $1.29 \pm 0.11 \mathrm{~b} \mathrm{BC}$ & $\underline{0.86 \pm 0.06 \mathrm{~b} \mathrm{C}}$ & $\underline{0.44 \pm 0.29 \mathrm{c} \mathrm{C}}$ \\
\hline & $\mathrm{P}$ & 0.991 & $0.004 * *$ & $0.001 * *$ & $0 * * *$ & $0 * * *$ \\
\hline \multirow{6}{*}{ BBCH68 } & $\mathrm{CK}$ & $5.12 \pm 1.00 \mathrm{~A}$ & $3.63 \pm 0.25 \mathrm{a} \mathrm{B}$ & $2.34 \pm 0.10$ a D & $1.96 \pm 0.14 \mathrm{C}$ & $2.15 \pm 0.48 \mathrm{C}$ \\
\hline & S4 & $5.32 \pm 1.21 \mathrm{~A}$ & $\underline{4.03 \pm 0.44 \mathrm{a} \mathrm{B}}$ & $\underline{2.53 \pm 0.12 \mathrm{a} \mathrm{C}}$ & $\underline{1.98 \pm 0.02 \mathrm{C}}$ & $2.16 \pm 0.21 \mathrm{C}$ \\
\hline & S3 & $4.95 \pm 0.71 \mathrm{~A}$ & $\underline{3.57 \pm 0.31 \mathrm{a} \mathrm{B}}$ & $\underline{2.69 \pm 0.10 \mathrm{a} \mathrm{C}}$ & $\underline{1.92 \pm 0.39 \mathrm{C}}$ & $2.02 \pm 0.37 \mathrm{C}$ \\
\hline & $\mathrm{S} 2$ & $\underline{4.51 \pm 0.40 \mathrm{~A}}$ & $2.62 \pm 0.07 \mathrm{~b} \mathrm{~B}$ & $\underline{2.37 \pm 0.38 \mathrm{a} \mathrm{BC}}$ & $\underline{1.93 \pm 0.27 \mathrm{C}}$ & $\underline{1.91 \pm 0.07 \mathrm{C}}$ \\
\hline & S1 & $\underline{3.59 \pm 0.32 \mathrm{~A}}$ & $2.14 \pm 0.16 \mathrm{~b} \mathrm{~B}$ & $1.77 \pm 0.35 \mathrm{~b} \mathrm{BC}$ & $\underline{1.68 \pm 0.68 \mathrm{BC}}$ & $\underline{1.39 \pm 0.16 \mathrm{C}}$ \\
\hline & $\mathrm{P}$ & 0.153 & $0 * * *$ & $0.01 * *$ & 0.866 & 0.055 \\
\hline \multirow{6}{*}{ BBCH75 } & CK & $3.67 \pm 1.87$ & $3.73 \pm 0.55$ & $3.54 \pm 0.39$ & $2.82 \pm 0.70$ & $2.66 \pm 1.09$ \\
\hline & S4 & $3.75 \pm 1.50$ & $\underline{3.72 \pm 0.23}$ & $\underline{3.32 \pm 0.24}$ & $\underline{2.82 \pm 0.19}$ & $2.58 \pm 0.74$ \\
\hline & S3 & $3.68 \pm 2.30$ & $\underline{3.62 \pm 0.03}$ & $\underline{3.21 \pm 0.05}$ & $\underline{2.78 \pm 0.03}$ & $\underline{2.67 \pm 0.30}$ \\
\hline & S2 & $\underline{3.72 \pm 2.08}$ & $\underline{3.59 \pm 0.27}$ & $\underline{3.05 \pm 0.23}$ & $\underline{2.96 \pm 0.61}$ & $\underline{2.63 \pm 0.25}$ \\
\hline & S1 & $\underline{3.66 \pm 2.51}$ & $\underline{3.55 \pm 0.40}$ & $\underline{2.98 \pm 0.59}$ & $\underline{2.65 \pm 0.12}$ & $\underline{2.43 \pm 0.24}$ \\
\hline & $\mathrm{P}$ & 0.998 & 0.952 & 0.369 & 0.934 & 0.989 \\
\hline
\end{tabular}

$\dagger$ Data followed by the same lowercase letter in a column represent nonsignificant differences among the treatments as determined through the least significant difference (LSD) test $(\mathrm{P}=0.05)$. Data followed by the same capital letter in a row represent nonsignificant differences among the diurnal varation of each treatment as determined through the least significant difference (LSD) test $(\mathrm{P}=0.05)$. The underlined number is the value under shading time. $\mathrm{CK}$ represents the "no shading" treatment (control), whereas S4, S3, S2, and S1 refer to the light saturation point (LSP) $+400 \mu \mathrm{mol} \mathrm{m}{ }^{-2} \mathrm{~s}^{-1}$, LSP $+100 \mu \mathrm{mol} \mathrm{m}^{-2} \mathrm{~s}^{-1}$, LSP $200 \mu \mathrm{mol} \mathrm{m} \mathrm{m}^{-2} \mathrm{~s}^{-1}$, and LSP $-500 \mu \mathrm{mol} \mathrm{m}^{-2} \mathrm{~s}^{-1}$, respectively. Data P-P value of shading treatment. *, significance at the .05 level; **, significance at the .01 level; ***, significance at the .001 level. Values are means \pm standard deviation of four independent replicates

The effect of shading on WUE ins of wheat functional leaves gradually weakened over the progression of a day. The periods of significant influence of shading during the $\mathrm{BBCH} 47, \mathrm{BBCH} 55, \mathrm{BBCH} 68$, and $\mathrm{BBCH} 75$ growth periods were 8:00-18:00, 10:0018:00, and 10:00-14:00, respectively (Table 3). The declines in WUE ins of S2 and S1 under shading during the corresponding periods were significantly different from that of the other treatments. The effects of shading on $\mathrm{WUE}_{\text {ins }}$ were different among the different growth stages. WUE ins gradually decreased with increased shading at $\mathrm{BBCH} 47$, with the increase in S4 and S3 at 12:00-14:00 during BBCH55, while that in $\mathrm{S} 4$ was at 8:00-18:00 and that at S3 and S2 were at 12:00-14:00 during BBCH68. The maximum value of $\mathrm{WUE}_{\text {ins }}$ was $5.32 \mu \mathrm{mol} \mathrm{CO} \mathrm{Col}^{-1} \mathrm{H}_{2} \mathrm{O}$ during $\mathrm{BBCH} 68$ at 8:0010:00 in S4. 
The impact of shading on the WUE int of functional leaves

The diurnal WUE $\mathrm{E}_{\text {int }}$ of $\mathrm{CK}$ during the different growth stages first increased and then decreased (Table 4). There were considerable changes to $\mathrm{WUE}_{\text {int }}$ during $\mathrm{BBCH} 47$ and $\mathrm{BBCH75}$, and the maximum change occurring at 14:00-16:00. There were relatively small changes in $\mathrm{WUE}_{\text {int }}$ during $\mathrm{BBCH} 55$ and $\mathrm{BBCH} 68$, with the maximum changes occurring at 10:00-12:00 and 12:00-14:00, respectively. The changes in WUE int were slightly different among the different treatments with increasing shading. There were consistent changes in $\mathrm{WUE}_{\text {int }}$ under $\mathrm{CK}$ and $\mathrm{S} 4$, whereas that of other treatments during BBCH47 gradually decreased after 12:00, and that of S1 and S2 during BBCH55 gradually decreased after 10:00. WUE int gradually increased during BBCH68 in S3 and $\mathrm{S} 4$, whereas that in $\mathrm{S} 1$ gradually decreased. The change in $\mathrm{WUE}_{\text {int }}$ in $\mathrm{BBCH} 75$ was consistent with that in CK.

Significant changes in daily $\mathrm{WUE}_{\text {int }}$ were concentrated during $\mathrm{BBCH} 47$ in all treatments besides for $\mathrm{S} 3$ and during $\mathrm{BBCH} 55$ besides for $\mathrm{S} 1$, with non-significant differences during the other growth stages.

With increased shading and wheat growth, WUE int gradually decreased during $\mathrm{BBCH} 47$, increased during BBCH55 in $\mathrm{S} 4$ at 10:00-14:00, increased during BBCH68 in S3 and S4 at all times, and increased during BBCH75 at all times except at 10:0012:00 (Table 4). The significant effect of shading on $\mathrm{WUE}_{\text {int }}$ was concentrated during $\mathrm{BBCH} 47$ and $\mathrm{BBCH} 55$ at 14:00-18:00 and B 12:00-18:00, respectively, and during both $\mathrm{BBCH} 68$ and $\mathrm{BBCH} 75$ at 10:00-12:00. There was a significant decrease in $W_{\text {W }}$ int $\mathrm{S} 1$ during these periods of significant effect, with the same pattern evident in S2 except during BBCH68.

Table 4. Effect of shading on intrinsic water use efficiency $\left(\mu \mathrm{mol} \mathrm{CO}_{2} \mathrm{~mol}^{-1} \mathrm{H}_{2} \mathrm{O}\right)$ during different growing stages in 2017

\begin{tabular}{|c|c|c|c|c|c|c|}
\hline Growth stage & Treatment & 8:00:00-10:00 & 10:00-12:00 & 12:00-14:00 & 14:00-16:00 & 16:00-18:00 \\
\hline \multirow{6}{*}{ ВBCH47 } & $\mathrm{CK}$ & $59.24 \pm 1.14 \mathrm{D}$ & $77.42 \pm 3.22 \mathrm{C}$ & $83.58 \pm 4.26 \mathrm{BC}$ & $103.16 \pm 7.42 \mathrm{aA} \dagger$ & $91.78 \pm 4.08 \mathrm{a} \mathrm{B}$ \\
\hline & S4 & $61.78 \pm 2.69 \mathrm{C}$ & $83.31 \pm 1.74 \mathrm{~B}$ & $77.14 \pm 5.83 \mathrm{~B}$ & $\underline{98.79 \pm 9.71 \mathrm{a} \mathrm{A}}$ & $83.17 \pm 5.55 \mathrm{~b} \mathrm{~B}$ \\
\hline & S3 & $58.63 \pm 0.29$ & $\underline{79.29 \pm 15.18}$ & $\underline{79.21 \pm 13.98}$ & $\underline{73.84 \pm 5.45 \mathrm{~b}}$ & $\underline{65.78 \pm 3.13 \mathrm{c}}$ \\
\hline & S2 & $\underline{54.90 \pm 2.03 \mathrm{BC}}$ & $\underline{72.40 \pm 8.80 \mathrm{~A}}$ & $\underline{68.36 \pm 9.20 \mathrm{AB}}$ & $\underline{63.11 \pm 11.92 \mathrm{bc} \mathrm{AB}}$ & $\underline{43.02 \pm 6.06 \mathrm{~d} \mathrm{C}}$ \\
\hline & S1 & $\underline{57.69 \pm 3.95 \mathrm{~A}}$ & $72.29 \pm 18.02 \mathrm{~A}$ & $\underline{65.65 \pm 16.22 \mathrm{~A}}$ & $\underline{52.39 \pm 3.76 \mathrm{c} \mathrm{A}}$ & $30.84 \pm 1.73 \mathrm{e} \mathrm{B}$ \\
\hline & $\mathrm{P}$ & 0.057 & 0.727 & 0.293 & $0 * * *$ & $0 * * *$ \\
\hline \multirow{6}{*}{ BBCH55 } & $\mathrm{CK}$ & $81.76 \pm 1.48$ & $86.42 \pm 6.86$ & $79.44 \pm 6.72 \mathrm{a}$ & $81.42 \pm 6.54 \mathrm{a}$ & $81.26 \pm 4.85 \mathrm{a}$ \\
\hline & S4 & $78.09 \pm 3.62$ & $\underline{88.39 \pm 6.93}$ & $\underline{82.76 \pm 10.23 \mathrm{a}}$ & $\underline{76.49 \pm 7.69 \mathrm{a}}$ & $71.09 \pm 3.45 \mathrm{a}$ \\
\hline & S3 & $81.76 \pm 16.99$ & $\overline{83.91 \pm 3.97}$ & $\overline{78.42 \pm 0.91 \mathrm{ab}}$ & $72.22 \pm 12.13 \mathrm{ab}$ & $\underline{68.31 \pm 11.81 \mathrm{a}}$ \\
\hline & S2 & $\underline{80.16 \pm 15.64}$ & $72.92 \pm 22.63$ & $\underline{67.42 \pm 6.91 \mathrm{bc}}$ & $\underline{57.76 \pm 9.49 \mathrm{~b}}$ & $\underline{45.76 \pm 11.87 \mathrm{~b}}$ \\
\hline & S1 & $\underline{71.26 \pm 9.05 \mathrm{~A}}$ & $\underline{63.06 \pm 11.03 \mathrm{~A}}$ & $\underline{62.24 \pm 3.73 \mathrm{c} \mathrm{A}}$ & $\underline{38.07 \pm 3.83 \mathrm{c} \mathrm{B}}$ & $\underline{21.36 \pm 12.78 \mathrm{c} \mathrm{C}}$ \\
\hline & $\mathrm{P}$ & 0.767 & 0.129 & $0.014 *$ & $0.001 * *$ & $0 * * *$ \\
\hline \multirow{6}{*}{ BBCH68 } & $\mathrm{CK}$ & $60.56 \pm 4.51$ & $66.32 \pm 2.82 \mathrm{a}$ & $68.70 \pm 5.01$ & $64.55 \pm 5.32$ & $66.29 \pm 17.91$ \\
\hline & S4 & $62.35 \pm 0.82$ & $\underline{67.16 \pm 1.79 \mathrm{a}}$ & $\underline{68.81 \pm 6.99}$ & $\underline{65.49 \pm 5.32}$ & $73.45 \pm 9.83$ \\
\hline & S3 & $59.76 \pm 4.12$ & $\overline{66.16 \pm 1.91 \mathrm{a}}$ & $\overline{69.32 \pm 5.18}$ & $71.82 \pm 16.76$ & $\underline{77.46 \pm 8.93}$ \\
\hline & S2 & $\underline{60.12 \pm 5.76}$ & $\underline{57.70 \pm 13.24 \mathrm{ab}}$ & $\underline{64.30 \pm 1.52}$ & $\underline{62.33 \pm 13.71}$ & $\underline{59.55 \pm 7.02}$ \\
\hline & $\mathrm{S} 1$ & $\underline{63.40 \pm 8.54}$ & $\underline{50.11 \pm 5.95 \mathrm{~b}}$ & $\underline{56.24 \pm 10.28}$ & $\underline{58.52 \pm 23.82}$ & $\underline{49.00 \pm 10.50}$ \\
\hline & $\mathrm{P}$ & 0.901 & $0.043 *$ & 0.141 & 0.854 & 0.076 \\
\hline \multirow{6}{*}{ BBCH75 } & $\mathrm{CK}$ & $50.82 \pm 26.99$ & $78.26 \pm 9.07 \mathrm{ab}$ & $79.91 \pm 8.41$ & $86.54 \pm 7.33$ & $75.61 \pm 26.65$ \\
\hline & S4 & $53.54 \pm 23.66$ & $\underline{85.20 \pm 2.09 \mathrm{a}}$ & $\underline{83.00 \pm 6.83}$ & $\underline{88.96 \pm 10.23}$ & $78.88 \pm 23.91$ \\
\hline & S3 & $52.75 \pm 34.22$ & $\overline{78.00 \pm 0.99 \mathrm{ab}}$ & $\overline{89.57 \pm 2.42}$ & $93.03 \pm 1.80$ & $\underline{87.02 \pm 4.57}$ \\
\hline & S2 & $\underline{54.01 \pm 29.56}$ & $71.98 \pm 5.40 \mathrm{~b}$ & $\underline{85.97 \pm 5.03}$ & $\underline{89.86 \pm 2.57}$ & $\underline{84.69 \pm 3.70}$ \\
\hline & S1 & $\underline{51.65 \pm 33.80}$ & $\underline{68.47 \pm 7.14 \mathrm{~b}}$ & $\underline{84.50 \pm 0.67}$ & $\underline{88.52 \pm 11.63}$ & $\underline{82.47 \pm 10.12}$ \\
\hline & $P$ & 0.996 & $0.042 *$ & 0.339 & 0.885 & 0.922 \\
\hline
\end{tabular}

$\dagger$ The definitions are as for Table 3 


\section{Effect of shading on grain yield, water consumption, and WUE}

There was a highly significant effect of shading and year on grain yield, with the interactions between shading and year significant (Table 5). The highest grain yield was obtained in S3, followed by S4, S2, CK, and S1. The variation in grain yield in response to shading was ascribed to the changes in kernel number per stem (KNPS) and spike number per hectare (SNPH). There were significant increases in KNPS in S4 and S3, whereas KNPS was reduced in S1. There were significant increases in SNPH in S4 and $\mathrm{S} 3$, whereas SNPH was reduced in S2 and S1. Also, thousand-kernel-weight (TKW) increased with increased shading.

Table 5. Effect of shading on spike number per hectare, kernel number per stem, thousandkernel weight (TKW), grain yield, total evapotranspiration (ET), and water use efficiency (WUE) during different growing stages

\begin{tabular}{|c|c|c|c|c|c|c|c|}
\hline Years & Treatment & $\begin{array}{l}\text { Spikes/ha } \\
\left(10^{4} \mathrm{ha}^{-1}\right)\end{array}$ & Kernels/spike & $\begin{array}{c}\text { TKW } \\
\text { (g) }\end{array}$ & $\begin{array}{l}\text { Grain yield } \\
\left(\mathrm{kg} \mathrm{ha}^{-1}\right)\end{array}$ & $\begin{array}{c}\mathbf{E T}_{\mathbf{t}} \\
(\mathbf{m m})\end{array}$ & $\begin{array}{c}\text { WUE } \\
\left(\mathrm{kg} \mathrm{ha}^{-1} \mathbf{m m}^{-1}\right)\end{array}$ \\
\hline \multirow{5}{*}{ 2016-2017 } & CK & $591.83 \pm 4.60 \mathrm{c} \dagger$ & $49.51 \pm 0.81 \mathrm{~b}$ & $45.71 \pm 0.16 \mathrm{c}$ & $\begin{array}{c}13394.79 \pm \\
285.24 \mathrm{~b}\end{array}$ & $629.52 \pm 4.76 \mathrm{ab}$ & $21.20 \pm 0.58 b c$ \\
\hline & $\mathrm{S} 4$ & $650.21 \pm 16.57 \mathrm{a}$ & $50.72 \pm 0.15 \mathrm{a}$ & $46.15 \pm 1.06 \mathrm{c}$ & $\begin{array}{c}15216.92 \pm \\
503.32 \mathrm{a}\end{array}$ & $670.82 \pm 14.20 \mathrm{a}$ & $21.21 \pm 0.65 \mathrm{bc}$ \\
\hline & S3 & $644.90 \pm 7.96 \mathrm{ab}$ & $50.79 \pm 0.73 \mathrm{a}$ & $47.69 \pm 0.56 b$ & $\begin{array}{c}15623.27 \pm \\
504.19 \mathrm{a}\end{array}$ & $628.91 \pm 12.05 \mathrm{ab}$ & $24.78 \pm 1.18 \mathrm{a}$ \\
\hline & $\mathrm{S} 2$ & $610.40 \pm 20.04 b c$ & $47.72 \pm 0.10 \mathrm{c}$ & $46.73 \pm 0.68 \mathrm{bc}$ & $\begin{array}{c}13608.15 \pm \\
298.79 b\end{array}$ & $607.28 \pm 50.17 b$ & $22.53 \pm 2.20 \mathrm{ab}$ \\
\hline & $\mathrm{S} 1$ & $514.86 \pm 33.15 \mathrm{~d}$ & $41.78 \pm 0.62 \mathrm{~d}$ & $53.46 \pm 0.32 \mathrm{a}$ & $\begin{array}{c}11492.65 \pm \\
575.35 \mathrm{c}\end{array}$ & $583.70 \pm 37.41 b$ & $19.77 \pm 1.98 \mathrm{c}$ \\
\hline \multirow{8}{*}{ 2017-2018 } & CK & $594.22 \pm 8.51 b$ & $48.33 \pm 0.58 \mathrm{~b}$ & $42.11 \pm 0.24 \mathrm{e}$ & $\begin{array}{c}12092.17 \pm \\
128.15 \mathrm{~d}\end{array}$ & $618.65 \pm 43.81 \mathrm{ab}$ & $19.60 \pm 1.17 \mathrm{c}$ \\
\hline & S4 & $633.33 \pm 1.53 \mathrm{a}$ & $49.53 \pm 0.06 \mathrm{a}$ & $43.36 \pm 0.32 \mathrm{~d}$ & $\begin{array}{c}13603.84 \pm \\
128.81 \mathrm{~b}\end{array}$ & $655.75 \pm 20.40 a$ & $20.76 \pm 0.63 b c$ \\
\hline & $\mathrm{S} 3$ & $637.08 \pm 2.60 \mathrm{a}$ & $50.33 \pm 0.58 \mathrm{a}$ & $44.53 \pm 0.22 b$ & $\begin{array}{c}14278.88 \pm \\
184.01 \mathrm{a}\end{array}$ & $612.69 \pm 22.30 \mathrm{ab}$ & $23.32 \pm 0.78 \mathrm{a}$ \\
\hline & $\mathrm{S} 2$ & $602.00 \pm 7.21 b$ & $47.17 \pm 0.29 \mathrm{c}$ & $43.92 \pm 0.14 \mathrm{c}$ & $\begin{array}{c}12469.95 \pm \\
183.65 \mathrm{c}\end{array}$ & $581.61 \pm 19.35 \mathrm{bc}$ & $21.45 \pm 0.59 \mathrm{~b}$ \\
\hline & $\mathrm{S} 1$ & $570.33 \pm 5.03 c$ & $33.67 \pm 0.58 \mathrm{~d}$ & $46.17 \pm 0.15 \mathrm{a}$ & $\begin{array}{c}8865.16 \pm \\
207.07 \mathrm{e}\end{array}$ & $544.86 \pm 26.39 \mathrm{c}$ & $16.31 \pm 1.13 \mathrm{~d}$ \\
\hline & Fs & $49.70 * * *$ & $624.41 * * *$ & $138.40 * * *$ & $179.59 * * *$ & $9.87 * * *$ & $19.30 * * *$ \\
\hline & Fy & 0.91 & $146.40 * * *$ & $511.60 * * *$ & $167.64 * * *$ & 3.83 & $13.98 * *$ \\
\hline & $F s \times y$ & $6.27 * *$ & $59.52 * * *$ & $24.05 * * *$ & $4.629 * *$ & 0.30 & 1.29 \\
\hline
\end{tabular}

$\dagger$ Data followed by the same letter in a column indicates a non-significant difference among the treatments according to the least significant difference (LSD) test $(\mathrm{P}<0.05)$. CK refers to the 'no shading' treatment (control), whereas S4, S3, S2, and S1, refer to light saturation point $(\mathrm{LSP})+400$, LSP +100 , LSP -200 , and LSP $-500 \mu \mathrm{mol} \mathrm{m} \mathrm{m}^{-2} \mathrm{~s}^{-1}$, respectively. Fs-F value of shading treatment, Fs-F value of year, Fs $\times$ y $-F$ value of interaction of shading by year. $*$, significance at the 0.05 level; **, significance at the 0.01 level; ***, significance at the 0.001 level. Values are means \pm standard deviation of four independent replicates

Only $\mathrm{ET}_{\mathrm{t}}$ was significantly affected by shading. While ET increased in $\mathrm{S} 4$, it decreased in S3, S2, and S1. The differences in ET were only significantly between S4 and $\mathrm{S} 1$ and between $\mathrm{S} 4$ and $\mathrm{S} 2$. The maximum $\mathrm{ET}_{\mathrm{t}}$ was in $\mathrm{S} 4$. The ET $\mathrm{T}_{\mathrm{t}}$ of $\mathrm{S} 4, \mathrm{~S} 3, \mathrm{~S} 2$, and $\mathrm{S} 1$ decreased by $-6.3,0.5,4.8$, and $9.6 \%$ compared to that in CK, respectively. WUE was significantly affected by shading and year, whereas it was not affected by the interaction of shading and year. The maximum WUE was observed in S3, followed by 
S2, S4, CK, and S1. WUE increased significantly in S3 but decreased in S1. The differences in WUE between CK and S4 and between S2 and S4 were not significant.

\section{Discussion}

\section{Grain yield response}

In contrast to that in other studies (Shen et al., 2020; Li et al., 2005), shading increased the yield of winter wheat in the present study. The increase in wheat yield was related to the degree of shading, with the yields of S4, S3, and S2 increasing with decreasing shading, while that of S1 decreased. The increase in yield observed in the treatments was not entirely due to the increase in Pn of functional leaves since Pn decreased with increased shading for all treatments except for S4. In S4, Pn increased during BBCH55, BBCH68, and BBCH75 (Fig. 4). However, photoprotection during high radiation hours resulted in the consumption of photosynthetic products (Kromdijk et al., 2016), whereas shading reduced the consumption of photosynthetic products. In addition, shading under strong light changed the quality of light and increased photosynthetic products of the lower leaves (Gago et al., 2014). Shading also promoted the conversion of photosynthetic products to yield indicators (Shen et al., 2020). At the same time, the improvement of photosynthesis during periods of low to moderate radiation weather (cloudy, rainy weather) through shading treatment is worthy of further research.

\section{Water use}

Consistent with Shen et al (2020), water consumption of winter wheat increased under increased shading. The intensity of water consumption in wheat was greatest during $\mathrm{BBCH} 65$, followed by $\mathrm{BBCH} 55, \mathrm{BBCH} 75$, and $\mathrm{BBCH} 47$ (Figs. 6, 7). The transpiration values of functional leaves under different treatments were consistent with whole barrel ET, in agreement with the results of previous studies (Shen et al., 2020). The Tr of functional leaves increased during BBCH75 in S4 (Fig. 6D). The ET of S4 increased from $\mathrm{BBCH} 68$ to $\mathrm{BBCH} 75$ compared to $\mathrm{CK}$ during typical sunny days, whereas that of the remaining treatments gradually decreased (Fig. 7). The similarity between the two scales was due to evapotranspiration in wheat being approximately negligible after the jointing stage (BBCH30) dominated by transpiration (Zheng, 2008). The two scales differed in that ET in S4 increased earlier than Tr, and the patterns of water consumption of shading treatments were different among the different growth stages. Primarily, functional leaf Tr represented the change in a single leaf (Fig. 6), whereas daily ET included the sum of leaves of each plant (Fig. 7). More effective spike plants under shading (Table 5) showed higher Tr.

The variations in $\mathrm{ET}_{\mathrm{t}}$ (Table 4) across the stages of wheat growth were the same as that of typical daily ET (Fig. 7) during BBCH55, BBCH68, and $\mathrm{BBCH} 75$, during which ET of S4 increased with increasing intensity of shading intensity, whereas it decreased within the other treatments. The reductions in $\mathrm{ET}_{\mathrm{t}}$ in $\mathrm{S} 3, \mathrm{~S} 2$, and $\mathrm{S} 1$ were $0.5,4.8$, and $9.6 \%$, respectively (Table 4), with these reductions significantly smaller than variations in ET during the different growth stages (Fig. 7). This difference was mainly observed during the unshaded growth period, including the period before jointing (accounted for $-5.4 \%$ of total water consumption), at night, and during the daylight hours of insufficient light (cloudy and rainy days). This indicates that the potential to save water increases with increasing effective shading. 


\section{WUE}

WUE represents the balance between gains in carbon and the associated water costs, and can be measured at different spatiotemporal scales (Gago et al., 2014). At the leaf level, $\mathrm{P}_{\mathrm{n}} / \mathrm{Tr}$ and $\mathrm{P}_{\mathrm{n}} / \mathrm{g}_{\mathrm{s}}$ are mostly used to characterize environmental and genetic effects, respectively. The present study determined leaf WUE using $\mathrm{P}_{\mathrm{n}}, \mathrm{g}_{\mathrm{s}}$, and Tr. There was a generally increasing and then decreasing trend in daily variation in $\mathrm{P}_{\mathrm{n}}$ in the CK treatment, with the maximum value at 10:00-12:00 (Fig. 4), The variation in $\operatorname{Tr}$ was in agreement with that in $\mathrm{P}_{\mathrm{n}}$, except for the maximum value of $\mathrm{Tr}$ being at 12:00-14:00 (Fig. 6), with $\mathrm{g}_{\mathrm{s}}$ showing a generally decreasing trend (Fig. 5). These results could mainly be attributed to environmental changes resulting from radiation and increases in temperature. The maximum value of daily radiation was at 12:00, whereas that of temperature was at 14:00, with the temperature beginning to exceed $30{ }^{\circ} \mathrm{C}$ at $13: 00$ (Table 2). Wheat experiences "midday depression" under high temperature and radiation (Figs. 4, 5, 6) (Shen et al., 2020). These three changes also lead to a gradual decrease in $\mathrm{WUE}_{\text {ins }}$ (Table 3) with a maximum WUE ins at 8:00-10:00, whereas WUE int gradually increased and then decreased (Table 4) with changes to the maximum period.

$\mathrm{P}_{\mathrm{n}}, \mathrm{Tr}$, and $\mathrm{g}_{\mathrm{s}}$ showed different changes under shading among different temporal scales, which changed the daily trend of each treatment. $\mathrm{WUE}_{\text {ins }}$ and $\mathrm{WUE}_{\text {int }}$ showed similar changes under shading, with both decreasing gradually during $\mathrm{BBCH} 47$. WUE $\mathrm{Wn}_{\text {ins }}$ and $\mathrm{WUE}_{\text {int }}$ increased in $\mathrm{S} 4$ at 12:00-14:00 at $\mathrm{BBCH} 55$, whereas that in $\mathrm{S} 4$ increased during $\mathrm{BBCH} 68$ and that in $\mathrm{S} 3$ increased during BBCH68. The maximum values of $W_{\text {Wins }}$ and $W_{\text {Unt }}$ gradually decreased with increasing shading during BBCH47. These patterns also indicated that wheat was significantly affected by shading during the early growth stages (Dong et al., 2014), the treatments were more sensitive to shading response, and that the effect of shading on leaf growth diminished with the adaptation to long-term shading during the later stages (Mo et al., 2015). Even though there was an increase in the differences in radiation between treatments (Fig. 1), and the intensity of natural radiation increased during the later stages.

At the level of each pot of wheat biota, WUE reflected the comprehensive spatial and temporal impacts on wheat, the formation of wheat community yield, and the use of water. Ensuring that a certain amount of water produced more yield requires a reduction in water consumption and an increase in yield. A reduction in water consumption along with an excessive reduction in yield will also lead to a reduction in WUE (Shen et al., 2020).

Shading showed different influences on WUE $\mathrm{W}_{\text {ins, }}$ WUE $\mathrm{E}_{\text {int, }}$ and WUE. WUE $\mathrm{E}_{\text {ins }}$ and $W_{U E} E_{\text {int }}$ were calculated based on the gas exchange parameters of functional leaves. As affected by both leaf growth and meteorological parameters, WUE ins and $W_{\text {UE }}$ changed dynamically. WUE was calculated by grain yield divided by water use. It was a comprehensive manifestation of the whole wheat community and the growth environment during the whole growth period. And WUE was a relatively fixed value after harvest.

\section{Conclusions}

The present study used saturated light intensity time-shading to obtain an increase in yield in a range that ensured that the maximum light intensity was slightly greater or less than the saturated light intensity (LSP $-200<$ radiation $<$ LSP $+400 \mu \mathrm{mol} \mathrm{m}{ }^{-2}$ $\mathrm{s}^{-1}$ ). The increased yield was a result of not only an increase in the photosynthetic rate 
of the functional leaves, but also as a result of the increase in the photosynthesis of the overall wheat community, including an increase in the number of effective spike plants and an increase in the leaf area index.

In addition, water consumption in winter wheat was reduced under saturation light intensity and below. Although the reduction in water consumption was not significant, WUE was improved and significant increases in yield and WUE were obtained at the maximum radiation of LSP $+400 \mu \mathrm{mol} \mathrm{m} \mathrm{m}^{-2} \mathrm{~s}^{-1}$. Water-saving potential in the high radiation area of Northwest China increased as the saturated light intensity of wheat fertility zone fell below $1400 \mu \mathrm{mol} \mathrm{m} \mathrm{m}^{-2} \mathrm{~s}^{-1}$. To fully clarify shading effects on wheat growth and water consumption, future studies should include the determination of photosynthesis and transpiration of other leaves besides functional leaves.

Acknowledgments. The project was supported by the National Natural Science Foundation of China (No. 51609197), CAS “Light of West China” Program (No. XAB2016AW06), Programme of Introducing Talents of Discipline to Universities (No. 104-451115012), and Scientific Research Program Funded by Shaanxi Provincial Education Department (No. 16JS084).

\section{REFERENCES}

[1] Acreche, M., Briceño-Felix, G., J. A., M., Slafer, G. (2009): Grain number determination in an old and a modern Mediterranean wheat as affected by pre-anthesis shading. - Crop and Pasture Science 60: 271.

[2] Araki, T., Kitano, M., Eguchi, H. (2000): Dynamics of fruit growth and photoassimilate translocation in tomato plant (Lycopersicon esculentum mill.) under controlled environment. - ISHS Acta Horticulturae 534: International Conference and British-Israeli Workshop on Greenhouse Techniques towards the 3rd Millennium, pp. 85-92. 10.17660/ActaHortic.2000.534.8.

[3] Bian, C., Ma, C., Liu, X., Gao, C., Liu, Q., Yan, Z., Ren, Y., Li, Q. (2016): Responses of winter wheat yield and water use efficiency to irrigation frequency and planting pattern.PloS ONE 11(5): e0124673.

[4] Chen, H., Li, Q.-P., Zeng, Y.-L., Deng, F., Ren, W.-J. (2019): Effect of different shading materials on grain yield and quality of rice. - Scientific Reports 9(1): 9992.

[5] de Freitas, S. T., Amarante, C. V. T. d., Dandekar, A. M., Mitcham, E. J. (2013): Shading affects flesh calcium uptake and concentration, bitter pit incidence and other fruit traits in 'Greensleeves' apple. - Scientia Horticulturae 161: 266-272.

[6] Deng, _F_, Wang, L., Yao, X., Wang, J. J., Yang, W. Y. (2009): Effects of differentgrowing-stage shading on rice grain-filling and yield. - Journal of Sichuan Agricultural University 27(3): 265-269.

[7] Dong, C., Fu, Y., Liu, G., Liu, H. (2014): Low light intensity effects on the growth, photosynthetic characteristics, antioxidant capacity, yield and quality of wheat (Triticum aestivum L.) at different growth stages in BLSS. - Advances in Space Research 53(11): 1557-1566.

[8] Dong, B., Yang, H., Liu, H., Qiao, Y., Zhang, M., Wang, Y., Xie, Z., Liu, M. (2019): Effects of shading stress on grain number, yield, and photosynthesis during early reproductive growth in wheat. - Crop Science 59(1): 363-378.

[9] Freitas, D., Tonetto, S., Shackel, A., K., Mitcham, J., E. (2011): Abscisic acid triggers whole-plant and fruit-specific mechanisms to increase fruit calcium uptake and prevent blossom end rot development in tomato fruit. - Journal of Experimental Botany 62(8): 2645-2656. 
[10] Gago, J., Douthe, C., Florez-Sarasa, I., Escalona, J. M., Galmes, J., Fernie, A. R., Flexas, J., Medrano, H. (2014): Opportunities for improving leaf water use efficiency under climate change conditions. - Plant Science: An International Journal of Experimental Plant Biology 226: 108-119.

[11] Gill, R. I. S., Singh, B., Kaur, N. (2009): Productivity and nutrient uptake of newly released wheat varieties at different sowing times under poplar plantation in northwestern India. - Agroforestry Systems 76(3): 579-590.

[12] Goto, N., Yamamoto, K. T., Watanabe, M. (1993): Action spectra for inhibition of hypocotyl growth of wild-type plants and of the hy2 long-hypocotyl mutant of Arabidopsis thaliana L. - Photochemistry and Photobiology 57(5): 867-871.

[13] Grashoff, C., d'Antuono, L. F. (1997): Effect of shading and nitrogen application on yield, grain size distribution and concentrations of nitrogen and water soluble carbohydrates in malting spring barley (Hordeum vulgare L.). - European Journal of Agronomy 6(3-4): 275-293.

[14] Ho, L., White, P. J. (2005): A cellular hypothesis for the induction of blossom-end rot in tomato fruit. - Annals of Botany 95(4): 571-581.

[15] Ili, Z. S., Fallik, E. (2017): Light quality manipulation improves vegetable quality at harvest and postharvest: a review. - Environmental and Experimental Botany 139: 79-90.

[16] Jin, N., Ren, W., Tao, B., He, L., Ren, Q., Li, S., Yu, Q. (2018): Effects of water stress on water use efficiency of irrigated and rainfed wheat in the Loess Plateau, China. - Science of the Total Environment 642: 1-11.

[17] Kromdijk, J., Głowacka, K., Leonelli, L., Gabilly, S. T., Iwai, M., Niyogi, K. K., Long, S. P. (2016): Improving photosynthesis and crop productivity by accelerating recovery from photoprotection. - Science (New York) 354(6314): 857-861.

[18] Li, C. H., Luan, L. M., Yin, F., Wang, Q., Zhao, Y. L. (2005): Effects of light stress at different stages on the growth and yield of different maize genotypes (Zea mays L.). Acta Ecologica Sinica 25(4): 824-830.

[19] Loveys, B. R., Düring, H. (1984): Diurnal changes in water relations and abscisic acid in field-grown Vitis vinifera cultivars. - The New Phytologist 97(1): 37-47.

[20] Mo, Z., Li, W., Pan, S., Fitzgerald, T. L., Xiao, F., Tang, Y., Wang, Y., Duan, M., Tian, H., Tang, X. (2015): Shading during the grain filling period increases 2-acetyl-1pyrroline content in fragrant rice. - Rice 8(1): 9.

[21] Mu, H., Jiang, D., Wollenweber, B., Dai, T., Jing, Q., Cao, W. (2010): Long-term low radiation decreases leaf photosynthesis, photochemical efficiency and grain yield in winter wheat. - Journal of Agronomy and Crop Science 196(1): 38-47.

[22] Ohashi-Kaneko, K., Matsuda, R., Goto, E., Fujiwara, K., Kurata, K. (2006): Growth of rice plants under red light with or without supplemental blue light. - Soil Science and Plant Nutrition 52(4): 444-452.

[23] Prioul, J. L., Chartier, P. (1977): Partitioning of transfer and carboxylation components of intracellular resistance to photosynthetic $\mathrm{CO} 2$ fixation: a critical analysis of the methods used. - Annals of Botany 4: 789-800.

[24] Ramanathan, V., Feng, Y. (2009): Air pollution, greenhouse gases and climate change: global and regional perspectives. - Atmospheric Environment 43(1): 37-50.

[25] Sandri, M. A., Andriolo, J. L., Witter, M., Dal Ross, T. (2003): Effect of shading on tomato plants grow under greenhouse. - Horticultura Brasileira 21(4): 642-645.

[26] Shahinnia, F., Le Roy, J., Laborde, B., Sznajder, B., Kalambettu, P., Mahjourimajd, S., Tilbrook, J., Fleury, D. (2016): Genetic association of stomatal traits and yield in wheat grown in low rainfall environments. - BMC Plant Biology 16(1): 150.

[27] Shen, Y., Zhang, J., Li, T. (2020): Effect of shading on the water use efficiency of winter wheat (Triticum aestivum L.) in semi-arid and semi-humid regions of China. - Applied Ecology and Environmental Research 18: 7739-7755.

[28] Taylor, M. D., Locascio, S. J. (2004): Blossom-end rot: a calcium deficiency. - Journal of Plant Nutrition 27(1): 123-139. 


$$
\text { - } 3321-
$$

[29] Thangaraj, M., Sivasubramanian, V. (1990): Effects of low light intensity on growth and productivity of irrigated rice (Oryza sativa L.). - Madras Agric J 77: 220-224.

[30] Wang, D. (2017): Water use efficiency and optimal supplemental irrigation in a high yield wheat field. - Field Crops Research 213(May): 213-220.

[31] Wang, R., Hua, C., Li, X., Zhang, Q., Jiao, D. (2005): Photosynthetic performance of PEPC transgenic rice under photoinhibition conditions. - Acta Agronomica Sinica 28(3): 321-326.

[32] Wang, Y., Zhang, Z., Liang, Y., Han, Yulong, Han, Yanlai, Bell, R. W. (2020): High potassium application rate increased grain yield of shading-stressed winter wheat by improving photosynthesis and photosynthate translocation. - Front. Plant Sci. https://doi.org/10.3389/fpls.2020.00134.

[33] Xu, C., Yin, Y., Cai, R., Wang, P., Ni, Y., Guo, J., Chen, E., Cai, T., Cui, Z., Liu, T., Yang, D., Wang, Z. (2013): Responses of photosynthetic characteristics and antioxidative metabolism in winter wheat to post-anthesis shading. - Photosynthetica 51(1): 139-150.

[34] Xu, C., Tao, H., Tian, B., Gao, Y., Ren, J., Wang, P. (2016): Limited-irrigation improves water use efficiency and soil reservoir capacity through regulating root and canopy growth of winter wheat. - Field Crops Research 196: 268-275.

[35] Zheng, C.-Y. (2008): Water consumption characteristic and dry matter accumulation and distribution in high-yielding wheat. - Acta Agronomica Sinica 34(8): 1450-1458. 